\title{
Perpendicular magnetism in magnetic multilayer systems
}

\author{
P. Weinberger ${ }^{a, b, *}$ L. Szunyogh ${ }^{a, c}$ \\ ${ }^{a}$ Center of Computational Materials Science, Karlsplatz 13, 1040 Wien, Vienna, Austria \\ ${ }^{\mathrm{b}}$ Institut für Elektrochemie und Festkörperchemie, TU Vienna, Vienna, Austria \\ ${ }^{\mathrm{c}}$ Institute of Theoretical Physics, TU Budapest, Budapest, Hungary
}

\begin{abstract}
The main aspects of the fully relativistic spin-polarized Screened Korringa-Kohn-Rostoker (KKR) method for layered systems (systems with only two-dimensional translational symmetry) are reviewed including, e.g., a discussion of the Dirac-Kohn-Sham Hamiltonian, magnetic configurations, the inhomogeneous coherent potential approximation (CPA) applicable to such systems, and a short description of the (classical) magnetic dipole-dipole interaction.

Based on this type of approach "perpendicular magnetism" is then discussed in terms of characteristic features frequently encountered in studies of the magnetic anisotropy energy of magnetic multilayer systems such as oscillations or reorientation transitions. For this purpose layer-resolved band energy contributions and their discrete Fourier transformations with respect to characteristic parameters are discussed for a few prominent systems. (C) 2000 Elsevier Science B.V. All rights reserved.
\end{abstract}

\section{Introduction}

In the last 5-10 years perpendicular magnetism became a very lively field of research, not only because of its technological implications reaching from magnetic recording to new kinds of computer memories, but also, and this in particular, because of inherent theoretical challenges: for the first time it seemed that relativistic effects lead to new technologies and technical devices.

In the present paper a brief summary of the Screened Korringa-Kohn-Rostoker (KKR) method as applied to layered systems (systems with only two-dimensional translational symmetry) is given. This method originated several years ago

\footnotetext{
${ }^{*}$ Corresponding author. Tel.: +43-1-58801-15830; fax: +43-158801-15899.

E-mail address: pw@cms.tuwien.ac.at (P. Weinberger).
}

from the Center for Computational Materials Science in Vienna and is now used in varying context in quite a few research institutions. Over the last few years, the fully relativistic spin-polarized version of this approach, applicable to magnetic multilayer systems, became a standard method for $a b$ initio calculations of perpendicular magnetism. It seems, therefore, that there is a definite need to summarize the main aspects of this kind of approach to magnetic anisotropy studies in multilayer systems. Consequently the first few sections in here deal with more formal aspects such as the properties of the Dirac-Kohn-Sham Hamiltonian, magnetic configurations, two-dimensional translational symmetry, etc., while in a section on applications mainly characteristic features of magnetic anisotropies in multilayer systems such as oscillations of the so-called band energy contribution to the magnetic anisotropy energy or reorientation transitions are illustrated. In there 
also a table of corresponding references is given, which eventually should be used for more detailed discussions and/or comparisons to experiment.

Although other, mostly semi-relativistic (use of the Pauli-Schrödinger equation) computational schemes (FP-LMTO, FLAPW, semi-empirical approaches) are nowadays in use for evaluating magnetic anisotropy quantities, in this article for matters of brevity no explicit reference is given to such studies. For the same reason also no discussion of phenomenological models (Landau-Lifshitz-type approaches) is included.

\section{Kohn-Sham-Dirac Hamiltonian}

In principle within the (non-relativistic) density functional theory (DFT) the Kohn-Sham-Dirac Hamiltonian is given by

$$
\mathscr{H}=\alpha \cdot \mathbf{p}+\beta m c^{2}+V^{\mathrm{eff}}[n, \mathbf{m}]+\beta \boldsymbol{\Sigma} \cdot \mathbf{B}^{\text {eff }}[n, \mathbf{m}],
$$

$\alpha_{i}=\left(\begin{array}{cc}0 & \sigma_{i} \\ \sigma_{i} & 0\end{array}\right), \quad \beta=\left(\begin{array}{cc}I_{2} & 0 \\ 0 & -I_{2}\end{array}\right)$,

$\Sigma_{i}=\left(\begin{array}{cc}\sigma_{i} & 0 \\ 0 & \sigma_{i}\end{array}\right), \quad I_{2}=\left(\begin{array}{cc}1 & 0 \\ 0 & 1\end{array}\right)$

$V^{\mathrm{eff}}[n, \mathbf{m}]=V^{\mathrm{ext}}+V^{\mathrm{Hartree}}+\frac{\delta E_{\mathrm{xc}}}{\delta n} \equiv V(\mathbf{r})$,

$\mathbf{B}^{\mathrm{eff}}[n, \mathbf{m}]=\mathbf{B}^{\mathrm{ext}}+\frac{e \hbar}{2 m c} \frac{\delta E_{\mathrm{xc}}}{\delta \mathbf{m}} \equiv \mathbf{B}(\mathbf{r})$,

where $n$ is the particle density, $\mathbf{m}$ the magnetization density, $V^{\text {eff }}[n, \mathbf{m}]$ the effective potential, $\mathbf{B}^{\text {eff }}[n, \mathbf{m}]$ the effective (exchange) magnetic field, $V^{\text {ext }}$ and $\mathbf{B}^{\text {ext }}$ the corresponding external fields, and $\alpha_{i}$ are Dirac and $\sigma_{i}$ Pauli (spin) matrices,

$$
\begin{aligned}
& \sigma_{x}=\left(\begin{array}{ll}
0 & 1 \\
1 & 0
\end{array}\right), \quad \sigma_{y}=\left(\begin{array}{cc}
0 & -i \\
i & 0
\end{array}\right), \\
& \sigma_{z}=\left(\begin{array}{cc}
1 & 0 \\
0 & -1
\end{array}\right), \\
& \alpha=\left(\alpha_{1}, \alpha_{2}, \alpha_{3}\right), \quad \sigma=\left(\sigma_{x}, \sigma_{y}, \sigma_{z}\right) .
\end{aligned}
$$

Consider now a rotation (point group operation) $R$, then invariance of $\mathscr{H}(\mathbf{r})$ by $R$ implies that

$S(R) \mathscr{H}\left(R^{-1} \mathbf{r}\right) S^{-1}(R)=\mathscr{H}(\mathbf{r})$,

where $S(R)$ is a $4 \times 4$ matrix transforming the Dirac matrices $\alpha_{i}, \beta$, and $\Sigma_{i}$

$S(R)=\left(\begin{array}{cc}U(R) & 0 \\ 0 & \operatorname{det}[ \pm] U(R)\end{array}\right)$,

and $U(R)$ is a (unimodular) $2 \times 2$ matrix and $\operatorname{det}[ \pm]=\operatorname{det}\left[D^{(3)}(R)\right]$ with $D^{(3)}(R)$ being the corresponding three-dimensional rotation matrix. Using the invariance condition in Eq. (8) explicitly, one can see immediately that the condition

$S(R)\left[I_{4} V\left(R^{-1} \mathbf{r}\right)\right] S^{-1}(R)=I_{4} V\left(R^{-1} \mathbf{r}\right)=I_{4} V(\mathbf{r})$

yields the usual (non-relativistic) rotational invariance condition for the potential $V(\mathbf{r})$, while the terms $S(R)[c \alpha \cdot \mathbf{p}] S^{-1}(R), \quad S(R)\left[\beta \mathbf{\Sigma} \cdot \mathbf{B}\left(R^{-1} \mathbf{r}\right)\right] S^{-1}(R)$,

have to be examined with more care. Considering the 'scalar product' in here term-wise, Eq. (11) reduces [15] to

$\mathbf{B}\left(R^{-1} \mathbf{r}\right)=\mathbf{B}(\mathbf{r}) \quad$ and $\quad U(R) \sigma U^{-1}(R)=\sigma$.

Consequently, the symmetry point group of $\mathscr{H}(\mathbf{r})$ is then defined as

$\mathscr{G}=\left\{R \mid S(R) \mathscr{H}\left(R^{-1} \mathbf{r}\right) S^{-1}(R)=\mathscr{H}(\mathbf{r})\right\}$.

\subsection{Kohn-Sham-Dirac Hamiltonian and the local density functional approximation}

Within the (non-relativistic) local density functional approximation (LDA) the Hamiltonian is usually defined as

$$
\begin{aligned}
& \mathscr{H}(\mathbf{r})=c \alpha \cdot \mathbf{p}+\beta m c^{2}+I_{4} V(\mathbf{r})+\beta \Sigma_{z} B(\mathbf{r}), \\
& V^{\mathrm{eff}}[n, \mathbf{m}] \equiv V(\mathbf{r}), \quad \mathbf{B}(\mathbf{r}) \equiv \mathbf{B}^{\mathrm{eff}}[n, \mathbf{m}] .
\end{aligned}
$$

Comparing with Eq. (1) the obvious meaning of the last term on the right-hand side of Eq. (14) is simply that the (effective) magnetization $\mathbf{B}(\mathbf{r})$ points along an arbitrary assumed $\hat{\mathbf{z}}$-direction, say $\mathbf{n} \in \mathscr{R}_{3}$, i.e., is of the form, 
$\mathbf{B}(\mathbf{r})=B(\mathbf{r}) \mathbf{n}, \quad \mathbf{n}=(0,0,1)$.

Suppose for matters of simplicity that

$V(\mathbf{r})=V(|\mathbf{r}|) \equiv V(r), \quad B(\mathbf{r})=B(|\mathbf{r}|) \equiv B(r)$,

then by inspecting rotational transformations of $\mathscr{H}(\mathbf{r})$, see Eq. (8), from Eqs. (10) and (11) it is obvious that any rotation $R$ leaves $V(r)$ and $B(r)$ invariant, whereas

$$
\begin{aligned}
U(R) \sigma_{z} B_{z}(r) U^{-1}(R) & =B(r) U(R)(\sigma \cdot \mathbf{n}) U^{-1}(R) \\
& =\left(\sigma^{\prime} \cdot \mathbf{n}\right),
\end{aligned}
$$

where

$\sigma^{\prime}=\left(\sigma_{x}^{\prime}, \sigma_{y}^{\prime}, \sigma_{z}^{\prime}\right)=U(R) \sigma U^{-1}(R)$.

Reviewing Eq. (18) again, it can be seen that the 'scalar product' on the right-hand side of this equation can also be interpreted as

$\left(\sigma^{\prime} \cdot \mathbf{n}\right)=(\sigma \cdot \xi)$

i.e., as if $\mathbf{B}(\mathbf{r})$ points along $\xi$,

$\mathbf{B}(\mathbf{r})=B(r) \xi$

$\xi=\operatorname{det}[ \pm] D^{(3)}(R) \mathbf{n}$.

Note that $\xi$ is a vector of unit length. Exactly this kind of "interpretation" is meant, when stating that $\mathbf{B}(\mathbf{r})$ is "orientated along a particular direction $\xi "[21]$.

\section{Two-dimensional translational invariance}

\subsection{Complex "square" lattices}

Suppose that the potential in Eq. (1), $V^{\text {eff }}[n, \mathbf{m}] \equiv V(\mathbf{r})$, is given as a superposition of individual ("non-overlapping") potentials ${ }^{1}$,

\footnotetext{
${ }^{1}$ The term non-overlapping potentials means that the domains of any given pair of potentials are disjoint in configuration space.
}

$$
\begin{aligned}
V(\mathbf{r})= & \sum_{s=1}^{S} \sum_{i} V\left(\left(r_{i, x}+R_{i, x}+c_{s, x}\right) \mathbf{x}\right. \\
& \left.+\left(r_{i, y}+R_{i, y}+c_{s, y}\right) \mathbf{y}+\left(r_{i, z}+R_{i, z}\right) \mathbf{z}\right), \\
= & \sum_{s=1}^{S} \sum_{i} V\left(\mathbf{r}_{i}+\mathbf{R}_{i, \|}+\mathbf{c}_{s, \|}+R_{i, z} \mathbf{z}\right) \\
\equiv & \sum_{s=1}^{S} \sum_{i} V_{i}^{s}\left(R_{i, z}\right),
\end{aligned}
$$

$(\mathbf{x}, \mathbf{x})=(\mathbf{y}, \mathbf{y})=(\mathbf{z}, \mathbf{z})=1$,

$(\mathbf{x}, \mathbf{y})=(\mathbf{x}, \mathbf{z})=(\mathbf{y}, \mathbf{z})=0$,

where the $\mathbf{c}_{s, \|}$ are the so-called non-primitive translations $\left(c_{s, z}=0 \forall s\right), S$ is the number of sublattices, the $\mathbf{R}_{i}$ refer to location vectors of Coulomb singularities in a particular sublattice, and $R_{i, z} \mathbf{Z}$ fixes the position of a plane of atoms in the configuration space $\Delta$. Quite clearly by considering the following translational invariance condition [15] in terms of function-space operators (denoted by a hat),

$$
\begin{aligned}
& \widehat{\mathbf{R}}_{j, \|} V\left(\mathbf{r}_{i}+\mathbf{R}_{i, \|}+R_{i, z} \mathbf{z}+\mathbf{c}_{s, \|}\right) \\
& \quad=V\left(\mathbf{r}_{i}+\mathbf{R}_{i, \|}-\mathbf{R}_{j, \|}+R_{i, z} \mathbf{z}+\mathbf{c}_{s, \|}\right),
\end{aligned}
$$

a complex two-dimensional lattice is defined by

$$
\begin{aligned}
\mathscr{L}\left(R_{i, z}\right) & =\left\{\mathbf{R}_{j, \|} \mid \widehat{\mathbf{R}}_{j, \|} V_{i}^{s}\left(R_{i, z}\right)=V_{i}^{s}\left(R_{i, z}\right)\right\} \\
& \equiv\left\{\mathbf{t}_{j, \|} \mid \mathbf{t}_{j, \|} V_{i}^{s}\left(R_{i, z}\right)=V_{i}^{s}\left(R_{i, z}\right)\right\},
\end{aligned}
$$

and the corresponding translational group as

$$
\mathscr{T}\left(R_{i, z}\right)=\left\{\left[E \mid \mathbf{t}_{i, \|}\right]\right\}, \quad\left[E \mid \mathbf{t}_{i, \|}\right]^{|\mathscr{T}|}=[E \mid \mathbf{0}],
$$

where $[E \mid \mathbf{0}]$ is the identity element, $E$ being the identity rotation and $|\mathscr{T}|$ denotes the group order. It should be noted that in (25) for a particular sublattice $s$ the "in-plane" site $\mathbf{R}_{i, \|}$ serves as the origin of $\mathscr{L}\left(R_{i, z}\right)$. A simple two-dimensional lattice refers then "simply" to the case of $S=1$ and $\mathbf{c}_{s, \|}=0$. 


\subsection{Multilayer systems}

Assuming now that in a multilayer system

$\mathscr{L}\left(R_{i, z}\right)=\mathscr{L}, \quad \mathscr{T}\left(R_{i, z}\right)=\mathscr{T} \quad \forall R_{i, z}$,

i.e., that in all layers one and the same two-dimensional translational symmetry applies, any arbitrary difference vector of $\mathbf{r} \in \Delta$ and $\mathbf{r}^{\prime} \in \Delta$ can be written as

$$
\begin{aligned}
\mathbf{r}-\mathbf{r}^{\prime}= & \mathbf{r}_{i}-\mathbf{r}_{j}+\mathbf{R}_{i, \|}-\mathbf{R}_{j, \|}+\mathbf{c}_{s, \|}-\mathbf{c}_{s^{\prime}, \|} \\
& +\left(c_{s, \perp}-c_{s^{\prime}, \perp}\right) \mathbf{z}, \\
= & \mathbf{r}_{i}-\mathbf{r}_{j}+\mathbf{R}_{i, \|}-\mathbf{R}_{j, \|}+\mathbf{c}_{s s^{\prime}},
\end{aligned}
$$

where in general $\left(c_{s, \perp}-c_{s^{\prime}, \perp}\right) \equiv\left(R_{i, z}-R_{j, z}\right)$, and

$$
\begin{aligned}
\mathbf{c}_{s s^{\prime}} & =\mathbf{c}_{s, \|}-\mathbf{c}_{s^{\prime}, \|}+\left(c_{s, \perp}-c_{s^{\prime}, \perp}\right) \mathbf{z} \\
& \equiv \mathbf{c}_{s s^{\prime}, \|}+c_{s s^{\prime}, \perp} \mathbf{z} .
\end{aligned}
$$

For a multilayer system corresponding to a simple two-dimensional lattice, the difference vector $\mathbf{c}_{s s^{\prime}}$ reduces to

$\mathbf{c}_{s s^{\prime}}=c_{s s^{\prime}, \perp} \mathbf{z}=\left(R_{i, z}-R_{j, z}\right) \mathbf{z}$.

It should be noted that Eq. (29) is the basis of the two-dimensional lattice Fourier transformations to be applied, since the projection operator with respect to the $\mathbf{k}_{\|}$-th irreducible representation of $\mathscr{T}$ is of the following form:

$$
\begin{aligned}
P_{\mathbf{k}_{\|}} & =|\mathscr{T}|^{-1} \sum_{\mathbf{R}_{\|} \in \mathscr{L}} \exp \left[-\mathrm{i}\left(\mathbf{k}_{\|} \cdot \mathbf{R}_{\|}\right)\right] \hat{\mathbf{R}}_{\|} \\
& =|\mathscr{T}|^{-1} \sum_{\left[E \mid \mathbf{t}_{\|}\right] \in \mathscr{T}} \exp \left[-\mathrm{i}\left(\mathbf{k}_{\|} \cdot \mathbf{t}_{\|}\right)\right]\left[E \mid \mathbf{t}_{i, \|}\right] .
\end{aligned}
$$

\subsection{Real and reciprocal two-dimensional lattices}

As is well known, the translations $\mathbf{t}_{i, \|} \in \mathscr{L}$ can be written as linear combinations of primitive twodimensional lattice vectors $\mathbf{a}_{1}$ and $\mathbf{a}_{2}$,

$\mathbf{t}_{i, \|} \equiv \mathbf{R}_{i, \|}=n_{1} \mathbf{a}_{1}+n_{2} \mathbf{a}_{2}$,

the (unit) surface area $A$ being defined as $A=\mathbf{a}_{1} \times \mathbf{a}_{2}$. The corresponding reciprocal lattice $\mathscr{L}^{-1}$ is then given by

$\mathscr{L}^{-1}=\left\{\mathbf{b}_{j, \|} \mid \mathbf{t}_{i, \|} \cdot \mathbf{b}_{j, \|}=2 \pi \delta_{i j} \forall \mathbf{t}_{i, \|} \in \mathscr{L}\right\}$, the unit area in $\mathscr{L}^{-1}$ being the unit area of the surface Brillouin zone. For matters of convenience in the following:

$I(\mathscr{L})=\left\{i \mid \mathbf{t}_{i, \|} V_{j}^{s}\left(\mathbf{r}_{j}\right)=V_{j}^{s}\left(\mathbf{r}_{j}\right)\right\}$

denotes the set of indices referring to $\mathscr{L}$ and

$I\left(\mathscr{L}^{-1}\right)=\left\{j \mid \mathbf{b}_{j, \|} \cdot \mathbf{t}_{i, \|}=2 \pi \delta_{i j} \forall \mathbf{t}_{i, \|} \in \mathscr{L}\right\}$

the corresponding set for $\mathscr{L}^{-1}$.

\section{Magnetic configurations}

Going now back to Eq. (14) and assuming that $B(\mathbf{r})$ is of the same form as $V(\mathbf{r})$, namely a superposition of individual (non-overlapping) effective exchange fields,

$$
\begin{aligned}
B(\mathbf{r}) & =\sum_{s=1}^{S} \sum_{i} B\left(\mathbf{r}_{i}+\mathbf{R}_{i, \|}+\mathbf{c}_{s, \|}+R_{i, z} \mathbf{z}\right) \\
& \equiv \sum_{s=1}^{S} \sum_{i} B_{i}^{S}\left(R_{i, z}\right) \xi_{i}^{S}\left(R_{i, z}\right),
\end{aligned}
$$

then translational invariance implies that in general, see also Eq. (26),

$$
\begin{aligned}
& \widehat{\mathbf{R}}_{j, \|} B_{i}^{s}\left(R_{i, z}\right)=B_{i}^{s}\left(R_{i, z}\right) \quad \text { and } \\
& \quad \forall i, j \in I\left(\mathscr{L}\left(R_{i, z}\right)\right),
\end{aligned}
$$

where $\xi_{0}^{s}\left(R_{i, z}\right)$ is the orientation of the magnetization in the origin of the sth sublattice in the plane of atoms characterized by $R_{i, z}$, i.e., within one particular (two-dimensional) sublattice the orientation of the magnetization has to be identical for all corresponding lattice points. This condition results directly from the fact that for translations the matrix $S(R)$ in Eq. (8) has to be a unit matrix. Quite clearly different sublattices and/or different planes may have different orientations.

Assuming now a (uniform) simple two-dimensional lattice $\mathscr{L}=\mathscr{L}\left(R_{i, z}\right) \forall R_{i, z}$, then in general a non-collinear arrangement of $N$ magnetic layers is defined by

$$
\begin{aligned}
\xi & =\left\{\xi_{0}\left(R_{1, z}\right), \xi_{0}\left(R_{2, z}\right), \ldots, \xi_{0}\left(R_{N, z}\right)\right\} \\
& \equiv\left\{\xi_{1}, \xi_{2}, \ldots, \xi_{N}\right\}
\end{aligned}
$$


where the $\xi_{i}$ are the unit vectors specifying the orientation of the magnetization in these layers. Suppose further one defines a reference configuration $\xi_{0} \equiv\left\{\xi_{k} \mid \xi_{k}=\xi_{0} \forall k\right\}$, where $\xi_{0}$ refers to a prechosen direction, and let $i$ denote the $i$ th layer in this system, then the (total) energy difference with respect to a change in the orientation of the magnetization in this particular layer, i.e., with respect to the configuration $\xi=\left\{\xi_{k} \mid \xi_{k}=\xi_{0}\right.$ $\forall k \neq i\}$, is given by

$\Delta E^{(N)}\left[\xi ; \xi_{0}\right]=E[\xi]-E\left[\xi_{0}\right]$.

For example, for an antiparallel orientation of the magnetization in layer $i, \xi_{i}=-\xi_{0}$, such an energy difference refers to a "spin-flip" energy. If simultaneously the orientation of the magnetization is changed in two (different) planes, say $i$ and $j$, i.e., by considering the configuration $\xi^{\prime}=\left\{\xi_{k} \mid \xi_{k}=\xi_{0}\right.$ $\forall k \neq i, j\}$, the corresponding energy difference is given by

$\Delta E^{(N)}\left[\xi^{\prime} ; \xi_{0}\right]=E\left[\xi^{\prime}\right]-E\left[\xi_{0}\right]$.

Quite clearly a "double spin-flip" energy, refers then to the case that in Eq. (39) $\xi_{i}=\xi_{j}=-\xi_{0}$. Choosing the reference configuration such that $\left(\xi_{0}, \hat{\mathbf{z}}\right)=1$, where $\hat{\mathbf{z}}$ refers to the surface normal, then the energy difference with respect to the magnetic configuration $\xi_{a} \equiv\left\{\xi_{k} \mid\left(\xi_{k}, \widehat{\mathbf{x}}\right)=1 \forall k\right\}$,

$\Delta E^{(N)}\left[\xi_{a} ; \xi_{0}\right]=E\left[\xi_{a}\right]-E\left[\xi_{0}\right]$

is traditionally called the (total) energy contribution to the perpendicular magnetic anisotropy energy. If on the other hand $\left(\xi_{0}, \hat{\mathbf{x}}\right)=1$, and $\xi_{a} \equiv\left\{\xi_{k} \mid \xi_{k}=\hat{\mathbf{y}} \forall k\right\}$, where $\hat{\mathbf{x}}$ and $\hat{\mathbf{y}}$ are in-plane unit vectors, $(\hat{\mathbf{x}}, \hat{\mathbf{y}})=0$, then the corresponding energy difference $\Delta E^{(N)}\left[\xi_{a} ; \xi_{0}\right]$ is called the (total) energy contribution to the in-plane magnetic anisotropy energy.

\section{Resolvents, Green's functions and scaling trans- formations}

Suppose the Hamilton operator $H$ (e.g., the Kohn-Sham-Dirac operator) is given in terms of an unperturbed Hamiltonian $H_{0}$ and a perturba- tion operator $V$, then clearly $H$ can immediately be written as

$H=H_{0}+V \equiv H_{0}+V+W-W \equiv H_{0}^{\prime}+V^{\prime}$,

where

$H_{0}^{\prime}=H_{0}-W, \quad V^{\prime}=V+W$.

As is well known, the resolvents of $H$ and $H_{0}$ are defined in the following manner:

$G(z)=(z-H)^{-1}, \quad G_{0}(z)=\left(z-H_{0}\right)^{-1}$,

from which readily the resolvent of $H_{0}^{\prime}$ follows:

$G_{0}^{\prime}(z)=\left(z-H_{0}^{\prime}\right)^{-1}=\left(z-H_{0}+W\right)^{-1}$,

or written in terms of a Dyson equation,

$G_{0}^{\prime}(z)=G_{0}(z)\left[1-W G_{0}^{\prime}(z)\right]$,

where $z=\epsilon+i \delta, \delta>0$, is a complex energy. From (43) and (44) then follows immediately that the Dyson equation for $G(z)$ can be written in terms of either $G_{0}(z)$ or $G_{0}^{\prime}(z)$

$G(z)=G_{0}(z)[1+V G(z)]=G_{0}^{\prime}(z)\left[1+V^{\prime} G(z)\right]$.

A Green's function is then the side-limit of a representation of $G(z)$ such as the below configuration space representations of $G^{0}(z)$ and $G(z)$,

$G^{0}\left(\mathbf{r}, \mathbf{r}^{\prime} ; \epsilon\right)=\lim _{\delta \rightarrow 0}\left\langle\mathbf{r}\left|G^{0}(z)\right| \mathbf{r}^{\prime}\right\rangle$,

$G\left(\mathbf{r}, \mathbf{r}^{\prime} ; \epsilon\right)=\lim _{\delta \rightarrow 0}\left\langle\mathbf{r}|G(z)| \mathbf{r}^{\prime}\right\rangle$.

\section{Screened KKR method}

\subsection{Real space structure constants}

Assuming for matters of simplicity that $\mathscr{L} \equiv \mathscr{L}\left(R_{i, z}\right) \forall R_{i, z}$, is a simple lattice $(S=1)$, and choosing $\mathbf{r}$ and $\mathbf{r}^{\prime}$ to be centered at (measured from) $\mathbf{R}_{i}=\mathbf{R}_{i, \|}+R_{i, z} \mathbf{z}$ and $\mathbf{R}_{j}=\mathbf{r}_{j}^{\prime}+\mathbf{R}_{j, \|}+R_{j, z} \mathbf{z}^{\prime}$, respectively, the configuration space representation of $G^{0}(z)$ in (50) easily can be switched to a "partial wave" representation, for details see e.g. [35], the matrix elements thereof being the socalled "real space structure constants", 
$\mathbf{G}^{0}(\epsilon)=\left\{G^{0, i j}(\epsilon)\right\}, \quad G^{0, i j}(\epsilon)=\left\{G_{L L^{\prime}}^{0, i j}(\epsilon)\right\}$,

where $L=(\ell m)$ refers to (non-relativistic) angular momentum indices.

\subsection{Muffin-tin geometries}

Furthermore, let us assume that the potentials in Eq. (23) and the exchange fields in Eq. (37) are of "muffin-tin type", i.e.,

$$
\begin{aligned}
V_{i}\left(R_{i, z}\right) & =V\left(\mathbf{r}_{i}+\mathbf{R}_{i, \|}+R_{i, z} \mathbf{z}\right) \\
& \equiv \begin{cases}V_{i}\left(\left|\mathbf{r}_{i}\right| ; R_{i, z}\right), & \left|\mathbf{r}_{i}\right| \leqslant b_{i}\left(R_{i, z}\right), \\
V_{0} & \text { otherwise, }\end{cases} \\
B_{i}\left(R_{i, z}\right) & =B\left(\mathbf{r}_{i}+\mathbf{R}_{i, \|}+R_{i, z} \mathbf{z}\right) \\
& \equiv \begin{cases}B_{i}\left(\left|\mathbf{r}_{i}\right| ; R_{i, z}\right), & \left|\mathbf{r}_{i}\right| \leqslant b_{i}\left(R_{i, z}\right), \\
B_{0} & \text { otherwise, }\end{cases}
\end{aligned}
$$

where the $b_{i}\left(R_{i, z}\right)$ are the so-called muffin-tin radii, $V_{0}$ and $B_{0}$ are the muffin-tin constants, and that the perturbation $W$ in Eq. (44) is also a superposition of individual (non-overlapping) potentials,

$W(\mathbf{r})=\sum_{i} W\left(\mathbf{r}_{i}+\mathbf{R}_{i, \|}+R_{i, z} \mathbf{z}\right),=\sum_{i} W_{i}\left(R_{i, z}\right)$,

with

$W_{i}\left(R_{i, z}\right)= \begin{cases}W_{r}, & \left|\mathbf{r}_{i}\right| \leqslant b_{i}\left(R_{i, z}\right) \\ V_{0} & \text { otherwise } \forall i \in I\left(\mathscr{L}\left(R_{i, z}\right)\right) \forall R_{i, z}\end{cases}$

where $W_{r}$ is a suitable constant, and $r$ stands for 'reference system'.

\subsection{Multiple scattering}

Using "traditional" multiple-scattering theory [34], the Green's function in Eq. (50) can be written in a partial wave representation as

$$
\begin{aligned}
& G\left(\mathbf{r}_{i}+\mathbf{R}_{i}, \mathbf{r}_{i}^{\prime}+\mathbf{R}_{j} ; \epsilon\right) \\
& =\sum_{L L^{\prime}} R_{L}^{i}\left(\mathbf{r}_{i} ; \epsilon\right) G_{L L^{\prime}}^{i j}(\epsilon) R_{L^{\prime}}^{j}\left(\mathbf{r}_{j}^{\prime}\right)+\delta_{i j} \\
& \quad \times \sum_{L} R_{L}^{i}\left(\mathbf{r}_{i,<} ; \epsilon\right) H_{L}^{i}\left(\mathbf{r}_{j,>} ; \epsilon\right),
\end{aligned}
$$

where the $R_{L}^{i}(\mathbf{r} ; \epsilon)$ and $H_{L}^{i}(\mathbf{r} ; \epsilon)$ are properly normalized regular and irregular scattering solutions
[35] corresponding to the energy $\epsilon$ and the potential $V_{i}\left(R_{i, z}\right)$. The so-called Green's function matrix $\mathbf{G}(\epsilon)=\left\{G^{i j}(\epsilon)\right\}, G^{i j}(\epsilon)=\left\{G_{L L^{\prime}}^{i j}(\epsilon)\right\}$ is then given by

$$
\begin{aligned}
\mathbf{G}(\epsilon)= & \mathbf{G}^{0}(\epsilon)+\mathbf{G}^{0}(\epsilon) \mathbf{t}(\epsilon) \mathbf{G}^{0}(\epsilon) \\
& +\mathbf{G}^{0}(\epsilon) \mathbf{t}(\epsilon) \mathbf{G}^{0}(\epsilon) \mathbf{t}(\epsilon) \mathbf{G}^{0}(\epsilon)+\cdots \\
= & \mathbf{G}^{0}(\epsilon)\left[\mathbf{I}-\mathbf{t}(\epsilon) \mathbf{G}^{0}(\epsilon)\right]^{-1},
\end{aligned}
$$

namely in terms of $\mathbf{G}^{0}(\epsilon)$, see Eq. (51), and of single-site $t$ matrices $\mathbf{t}(\epsilon)=\left\{t_{L}^{i}(\epsilon) \delta_{i j}\right\}$, with $\mathbf{I}$ denoting a unit matrix. The scattering-path operator $\tau(\epsilon)=\left\{\tau_{L L^{\prime}}^{i j}(\epsilon)\right\}$, see for example [35],

$\tau(\epsilon)=\left[\mathbf{t}(\epsilon)^{-1}-\mathbf{G}^{0}(\epsilon)\right]^{-1}$

is related to (57) through

$$
\begin{aligned}
\mathbf{G}(\epsilon) & =\mathbf{G}^{0}(\epsilon)+\mathbf{G}^{0}(\epsilon) \tau(\epsilon) \mathbf{G}^{0}(\epsilon) \\
& =\mathbf{t}(\epsilon)^{-1} \tau(\epsilon) \mathbf{t}(\epsilon)^{-1}-\mathbf{t}(\epsilon)^{-1}
\end{aligned}
$$

\subsection{Screening transformations}

Eqs. (57)-(59) can easily be reformulated with respect to a new reference system $r$. If the singlesite $t$ matrices corresponding to $W_{r}$ are denoted by $\mathbf{t}^{r}(\epsilon)$, the respective Green's function matrix, $\mathbf{G}^{r}(\epsilon)=\left\{G^{r, i j}(\epsilon)\right\}, \quad G^{r, i j}(\epsilon)=\left\{G_{L L^{\prime}}^{r, i j}(\epsilon)\right\}, \quad$ see also Eq. (46), is given by:

$$
\begin{aligned}
\mathbf{G}^{r}(\epsilon)= & \mathbf{G}^{0}(\epsilon)+\mathbf{G}^{0}(\epsilon) \mathbf{t}^{r}(\epsilon) \mathbf{G}^{0}(\epsilon) \\
& +\mathbf{G}^{0}(\epsilon) \mathbf{t}^{r}(\epsilon) \mathbf{G}^{0}(\epsilon) \mathbf{t}^{r}(\epsilon) \mathbf{G}^{0}(\epsilon)+\cdots, \\
= & \mathbf{G}^{0}(\epsilon)\left[\mathbf{I}-\mathbf{t}^{r}(\epsilon) \mathbf{G}^{0}(\epsilon)\right]^{-1} .
\end{aligned}
$$

By introducing the following difference,

$\mathbf{t}_{\Delta}(\epsilon)=\mathbf{t}(\epsilon)-\mathbf{t}^{r}(\epsilon)$

one obtains for $\mathbf{G}(\epsilon)$ :

$$
\begin{aligned}
\mathbf{G}(\epsilon)= & \mathbf{G}^{r}(\epsilon)+\mathbf{G}^{r}(\epsilon) \mathbf{t}_{\Delta}(\epsilon) \mathbf{G}^{r}(\epsilon) \\
& +\mathbf{G}^{r}(\epsilon) \mathbf{t}_{\Delta}(\epsilon) \mathbf{G}^{r}(\epsilon) \mathbf{t}_{\Delta}(\epsilon) \mathbf{G}^{r}(\epsilon)+\cdots, \\
= & \mathbf{G}^{r}(\epsilon)\left[\mathbf{I}-\mathbf{t}_{\Delta}(\epsilon) \mathbf{G}^{r}(\epsilon)\right]^{-1} .
\end{aligned}
$$

Defining finally the following scattering-path operator, 
$\tau_{\Delta}(\epsilon)=\left[\mathbf{t}_{\Delta}(\epsilon)^{-1}-\mathbf{G}^{r}(\epsilon)\right]^{-1}$,

$\mathbf{G}(\epsilon)$ can also be expressed as

$$
\begin{aligned}
\mathbf{G}(\epsilon) & =\mathbf{G}^{r}(\epsilon)+\mathbf{G}^{r}(\epsilon) \tau_{\Delta}(\epsilon) \mathbf{G}^{r}(\epsilon) \\
& =\mathbf{t}_{\Delta}(\epsilon)^{-1} \tau_{\Delta}(\epsilon) \mathbf{t}_{\Delta}(\epsilon)^{-1}-\mathbf{t}_{\Delta}(\epsilon)^{-1} .
\end{aligned}
$$

Therefore, once $\mathbf{t}^{r}(\epsilon)$ and $\mathbf{G}^{r}(\epsilon)$ are known, Eqs. (62)-(64) represent a set of equations equivalent to Eqs. (57)-(59). Combining Eq. (59) with Eq. (64) the below relation can easily be read off,

$$
\begin{aligned}
\tau(\epsilon)= & \mathbf{t}(\epsilon)\left[\mathbf{t}_{\Delta}(\epsilon)^{-1} \tau_{\Delta}(\epsilon) \mathbf{t}_{\Delta}(\epsilon)^{-1}\right. \\
& \left.+\left(\mathbf{t}(\epsilon)^{-1}-\mathbf{t}_{\Delta}(\epsilon)^{-1}\right)\right] \mathbf{t}(\epsilon) .
\end{aligned}
$$

\subsection{Screened structure constants}

By choosing a suitable $W_{r}$ Eq. (60) can be solved such that

$G_{\mathrm{NR}}^{r, i j}(\epsilon) \equiv G^{r, i j}(\epsilon) \sim 0 \quad$ for $\forall\left|\mathbf{R}_{i}-\mathbf{R}_{i}\right| \geqslant d$,

where the index NR serves as a reminder that $\mathbf{G}^{r}(\epsilon)$ refers to a non-relativistic partial wave representation and the distance $d$ has to be viewed as the radius of a sphere that comprises only a few types of "neighboring" sites such as first- and second-nearest neighbors $[9,17]$.

The so-called (non-relativistic) screened structure constants $\mathbf{G}^{r}(\epsilon)$ can then easily be related to their relativistic counterparts by means of the following transformation with Clebsch-Gordan coefficients $\mathscr{C}(\sigma)$, for details see Ref. [7],

$G_{\mathrm{R}}^{r, i j}(\epsilon)=\sum_{\sigma= \pm 1 / 2} \mathscr{C}(\sigma)^{\dagger} G_{N R}^{r, i j}(\epsilon) \mathscr{C}(\sigma)$

\subsection{Application to systems with two-dimensional translational invariance}

Suppose now that in all atomic layers one and the same two-dimensional translational symmetry applies, i.e., $\mathscr{L}\left(R_{i, z}\right)=\mathscr{L} \forall R_{i, z}$, see also Eq. (28), with $\mathscr{L}$ referring to a simple two-dimensional lattice, and - in order to simplify the notation - position vectors are simply denoted by $\mathbf{R}_{p i}$
$\mathbf{R}_{p i}=\mathbf{C}_{p}+\mathbf{R}_{i, \|}, \quad \mathbf{R}_{i, \|} \in \mathscr{L}, \quad \mathbf{C}_{p}=R_{i, z} \mathbf{z}$

where $\mathbf{C}_{p}$ is sometimes referred to as the "spanning vector" of a particular layer $p$. According to Eq. (66) for the lattice Fourier transformed screened structure constants,

$$
\begin{aligned}
G^{r, p q}\left(\mathbf{k}_{\|} ; \epsilon\right)= & \sum_{\mathbf{R}_{\|} \in \mathscr{L}} \exp \left[i \mathbf{k}_{\|} \cdot \mathbf{R}_{\|}\right] G^{r}\left(\mathbf{C}_{p}+\mathbf{R}_{\|}, \mathbf{C}_{q} ; \epsilon\right), \\
& p, q=1, \ldots, n,
\end{aligned}
$$

therefore the following assumption can be made:

$G^{r, p q}\left(\mathbf{k}_{\|} ; \epsilon\right)=0 \quad$ if $|p-q|>N$

where $N$ is a suitably chosen parameter. Consider now the following tridiagonal supermatrix,

$$
\left(\begin{array}{ccccccc}
0 & 0 & 0 & 0 & 0 & 0 & 0 \\
0 & \underline{A}_{21} & \underline{A}_{22} & \underline{A}_{23} & 0 & 0 & 0 \\
0 & 0 & \underline{A}_{32} & \underline{A}_{33} & \underline{A}_{34} & 0 & 0 \\
0 & 0 & 0 & \underline{A}_{43} & \underline{A}_{44} & \underline{A}_{45} & 0 \\
\vdots & & & & & \ddots &
\end{array}\right),
$$

i.e., a matrix where each matrix element $\underline{A}_{i j}$ is a square matrix. According to Eq. (70), a non-vanishing block of dimension Nof elements $G^{r, p q}\left(\mathbf{k}_{\|} ; \epsilon\right)$, $(|p-q| \leqslant N)$, can be viewed as one particular element of such a tridiagonal matrix. Labelling the rows and columns of this tridiagonal matrix by $P$ and $Q$ ("principal layers") the supermatrix of the screened structure constants, $\quad \mathbf{G}^{r}\left(\mathbf{k}_{\|} ; \epsilon\right)=$ $\left\{G^{r, p q}\left(\mathbf{k}_{\|} ; \epsilon\right)\right\}$, can therefore be viewed also as a tridiagonal matrix in principal layers, ${ }^{2}$

$\mathbf{G}^{r}\left(\mathbf{k}_{\|} ; \epsilon\right)=\left\{\underline{G}^{r, P Q}\left(\mathbf{k}_{\|} ; \epsilon\right)\right\}$.

Furthermore, if a parent three-dimensional lattice [15] can be assumed, i.e., if all interlayer distances are equal (no layer relaxation), then obviously the elements of this tridiagonal matrix are of the following form:

\footnotetext{
${ }^{2}$ Note that for matters of transparency in the following the elements of a matrix labelled by principle layers are characterized by underlined letters.
} 
$\underline{G}^{r, P Q}\left(\mathbf{k}_{\|} ; \epsilon\right)= \begin{cases}\underline{G}^{r, 00}\left(\mathbf{k}_{\|} ; \epsilon\right), & P=Q, \\ \underline{G}^{r, 01}\left(\mathbf{k}_{\|} ; \epsilon\right), & P=Q-1, \\ \underline{G}^{r, 10}\left(\mathbf{k}_{\|} ; \epsilon\right), & P=Q+1, \\ 0 & \text { otherwise. }\end{cases}$

It should be noted that in the case of layer relaxations in principle all $\underline{G}^{r}, P Q\left(\mathbf{k}_{\|} ; \epsilon\right)$ are different, see also [28], although $\mathbf{G}^{r}\left(\mathbf{k}_{\|} ; \epsilon\right)$ is still formally tridiagonal.

The $\mathbf{k}_{\|}$th projection of $\tau_{\Delta}(\epsilon)$, see Eq. (63), is then given by

$\tau_{\Delta}\left(\mathbf{k}_{\|} ; \epsilon\right)=\left[\mathbf{t}_{\Delta}(\epsilon)^{-1}-\mathbf{G}^{r}\left(\mathbf{k}_{\|} ; \epsilon\right)\right]^{-1}$

where

$\mathbf{t}_{\Delta}(\epsilon)=\left\{\underline{t}_{\Delta}^{P}(\epsilon) \delta_{P Q}\right\}, \quad \underline{t}_{\Delta}^{P}(\epsilon)=\left\{t_{\Delta}^{p}(\epsilon) \delta_{p q}\right\}$,

$\tau_{\Delta}\left(\mathbf{k}_{\|} ; \epsilon\right)=\left\{\underline{\tau}_{\Delta}^{P Q}\left(\mathbf{k}_{\|} ; \epsilon\right)\right\}, \quad \underline{\tau}_{\Delta}^{P Q}(\epsilon)=\left\{\tau_{\Delta}^{p q}(\epsilon)\right\}$.

Quite clearly, since $\mathbf{t}_{\Delta}(\epsilon)$ formally is a diagonal supermatrix, the inverse of $\tau_{\Delta}\left(\mathbf{k}_{\|} ; \epsilon\right)$ is of tridiagonal form.

Usually for a system with a surface or with interfaces, three regions of different physical properties can be distinguished, namely a left semiinfinite system $(\mathrm{L})$, a right semi-infinite system $(\mathrm{R})$ and an intermediate region (I), see also the discussion in $[15,36]$. These regions correspond to the following numbering scheme for principal layers:

$\mathrm{L}: \quad-\infty<P \leqslant 0$,

I : $\quad 1 \leqslant P \leqslant n$,

$\mathrm{R}: \quad n+1 \leqslant P<\infty$,

which in turn implies that $\left[\tau_{\Delta}\left(\mathbf{k}_{\|} ; \epsilon\right)\right]^{-1}$ can be partitioned as follows:

$$
\begin{aligned}
& {\left[\tau_{\Delta}\left(\mathbf{k}_{\|} ; \epsilon\right)\right]^{-1}} \\
& =\left(\begin{array}{ccc}
{\left[\tau_{\Delta}\left(\mathbf{k}_{\|} ; \epsilon\right)\right]_{\mathrm{L}, \mathrm{L}}^{-1}} & {\left[\tau_{\Delta}\left(\mathbf{k}_{\|} ; \epsilon\right)\right]_{\mathrm{L}, \mathrm{I}}^{-1}} & \mathbf{0} \\
{\left[\tau_{\Delta}\left(\mathbf{k}_{\|} ; \epsilon\right)\right]_{\mathrm{I}, \mathrm{L}}^{-1}} & {\left[\tau_{\Delta}\left(\mathbf{k}_{\|} ; \epsilon\right)\right]_{\mathrm{I}, \mathrm{I}}^{-1}} & {\left[\tau_{\Delta}\left(\mathbf{k}_{\|} ; \epsilon\right)\right]_{\mathrm{I}, \mathrm{R}}^{-1}} \\
\mathbf{0} & {\left[\tau_{\Delta}\left(\mathbf{k}_{\|} ; \epsilon\right)\right]_{\mathrm{R}, \mathrm{I}}^{-1}} & {\left[\tau_{\Delta}\left(\mathbf{k}_{\|} ; \epsilon\right)\right]_{\mathrm{R}, \mathrm{R}}^{-1}}
\end{array}\right) .
\end{aligned}
$$

In order to evaluate $\tau\left(\mathbf{k}_{\|} ; \epsilon\right)_{\mathrm{I}, \mathrm{I}}$ use can be made of the so-called surface scattering path operators, see, in particular, the discussion in [4,6], which in turn refer to the so-called "missing elements" in the above tridiagonal matrix,

$$
\begin{aligned}
\underline{\Delta}_{\mathrm{L}}\left(\mathbf{k}_{\|} ; \epsilon\right)= & {\left[\underline{t}_{\Delta}^{\mathrm{L}}(\epsilon)^{-1}-\underline{G}^{r, 00}\left(\mathbf{k}_{\|} ; \epsilon\right)\right.} \\
& \left.-\underline{G}^{r, 10}\left(\mathbf{k}_{\|} ; \epsilon\right) \underline{\Delta}_{\mathrm{L}}\left(\mathbf{k}_{\|} ; \epsilon\right) \underline{G}^{r, 01}\left(\mathbf{k}_{\|} ; \epsilon\right)\right]^{-1}, \\
\underline{\Delta}_{\mathrm{R}}\left(\mathbf{k}_{\|} ; \epsilon\right)= & {\left[\underline{t}_{\Delta}^{\mathrm{R}}(\epsilon)^{-1}-\underline{G}^{r, 00}\left(\mathbf{k}_{\|} ; \epsilon\right)\right.} \\
& \left.-\underline{G}^{r, 01}\left(\mathbf{k}_{\|} ; \epsilon\right) \underline{\Delta}_{\mathrm{R}}\left(\mathbf{k}_{\|} ; \epsilon\right) \underline{G}^{r, 10}\left(\mathbf{k}_{\|} ; \epsilon\right)\right]^{-1} .
\end{aligned}
$$

It should be noted that in Eqs. (79) and (80) and in the following equation a parent three-dimensional lattice is assumed. In terms of these two quantities, which have to be calculated self-consistently, the $P Q$ th element of the scattering path operator in the interface region is then given by

$$
\begin{aligned}
& {\left[\left[\tau_{\Delta}\left(\mathbf{k}_{\|} ; \epsilon\right)\right]_{\mathrm{I}, \mathrm{I}}^{-1}\right]^{P Q}} \\
& =\left(\underline{t}_{\Delta}^{P}(\epsilon)^{-1}-\underline{G}^{r, 00}\left(\mathbf{k}_{\|} ; \epsilon\right)\right) \delta_{P Q} \\
& \quad-\underline{G}^{r, 01}\left(\mathbf{k}_{\|} ; \epsilon\right) \delta_{P, Q-1}-\underline{G}^{r, 10}\left(\mathbf{k}_{\|} ; \epsilon\right) \delta_{P, Q+1} \\
& \quad-\underline{G}^{r, 10}\left(\mathbf{k}_{\|} ; \epsilon\right) \underline{\Delta}_{\mathrm{L}}^{r}\left(\mathbf{k}_{\|} ; \epsilon\right) \underline{G}^{r, 01}\left(\mathbf{k}_{\|} ; \epsilon\right) \delta_{P, 1} \delta_{Q, 1} \\
& \quad-\underline{G}^{r, 01}\left(\mathbf{k}_{\|} ; \epsilon\right) \underline{\Delta}_{\mathrm{R}}^{r}\left(\mathbf{k}_{\|} ; \epsilon\right) \underline{G}^{r, 10}\left(\mathbf{k}_{\|} ; \epsilon\right) \delta_{P, n} \delta_{Q, n} .
\end{aligned}
$$

Finally, with respect to two given sites, $\mathbf{R}_{n}=\mathbf{R}_{n, \|}+$ $\mathbf{C}_{p}$ and $\mathbf{R}_{m}=\mathbf{R}_{m, \|}+\mathbf{C}_{q}$, respectively, $\mathbf{R}_{n, \|}, \mathbf{R}_{m, \|} \in \mathscr{L}$, the so-called site representation of $\tau_{\Delta}\left(\mathbf{k}_{\|} ; \epsilon\right)$ in the interface region can be obtained by means of the following Surface Brillouin Zone (SBZ) integral,

$$
\begin{aligned}
\tau_{\Delta}^{n m}(\varepsilon)= & \frac{1}{\Omega_{\mathrm{SBZ}}} \int \exp \left[-\mathrm{i} \mathbf{k}_{\|} \cdot\left(\mathbf{R}_{n, \|}-\mathbf{R}_{m, \|}\right)\right] \\
& \times \tau_{\Delta}^{p q}\left(\mathbf{k}_{\|} ; \epsilon\right) \mathrm{d} \mathbf{k}_{\|},
\end{aligned}
$$

where $\Omega_{\mathrm{SBZ}}$ is the unit area of the two-dimensional SBZ. Since the "unscreened" scattering path operator $\tau^{n m}(\varepsilon)$ is always related to $\tau_{\Delta}^{n m}(\varepsilon)$ via Eq. (65), in the following sections simply $\tau^{n m}(\varepsilon)$ is used.

\subsection{Rotation of frames}

Now we shall go back to the Kohn-ShamDirac Hamiltonian in Eq. (14) and the section that 
introduced the concept of magnetic configurations. Let $R \in \mathrm{O}(3)$ be a rotation, which transforms the orientation of the effective magnetization $\xi^{p i}$ of site $i$ in the $p$ th layer into the direction $\widehat{\mathbf{z}}$. Furthermore, let $t^{p i}(\epsilon)$ refer to the single-site $t$-matrix if $\xi^{p i}$ is parallel to $\hat{\mathbf{z}}$, while $t_{R}^{p i}(\epsilon)$ refers to the $t$-matrix if $\xi^{p i}$ points along the direction $R^{-1} \widehat{\mathbf{z}}$. Because the effective potential and the effective exchange field are spherical symmetric, see Eqs. (52) and (53), these two single-site $t$-matrices are related to each other by the following similarity transformation,

$t_{R}^{p i}(\epsilon)=D(R) t^{p i}(\epsilon) D(R)^{+}$,

where $D(R)$ contains blockwise the irreducible projective representations [41] of $R$. Clearly enough, two-dimensional translational invariance then implies that

$t_{R}^{p i}(\epsilon)=t_{R}^{p 0}(\epsilon) \quad \forall i \in I(\mathscr{L})$,

where $i=0$ refers to the origin of $\mathscr{L}$.

Special care has to be taken in performing the occurring Brillouin Zone integrals needed to evaluate the elements of the scattering path operator, see Eq. (82), since in the presence of a magnetic field these integrals can no longer be restricted [3] to an irreducible wedge of the corresponding SBZ. Let $G$ be the point group of the underlying two-dimensional lattice, such as for example $C_{4 v}$ in the case of an fcc $\left(\begin{array}{lll}0 & 0 & 1\end{array}\right)$ surface, and suppose $D(S)$ contains blockwise the irreducible projective representations [41] of $S \in G$. If $\mathrm{IBZ}_{1}$ denotes an irreducible wedge of the SBZ, then any other wedge $\mathrm{IBZ}_{S}$ of the SBZ is defined by

$\mathrm{IBZ}_{S}=\left\{S \mathbf{k}_{\|} \mid \mathbf{k}_{\|} \in \mathrm{IBZ}_{1}\right\}, \quad S \in G$,

such that

$\mathrm{SBZ}=\sum_{S \in G} \mathrm{IBZ}_{S}$.

It can easily be shown that

$\tau^{p q}\left(S^{-1} \mathbf{k}_{\|} ; \epsilon\right)=D(S)^{+} \tau_{S}^{p q}\left(\mathbf{k}_{\|} ; \epsilon\right) D(S)$,

where - as should be noted $-p, q$ are layer indices and $\tau_{S}^{p q}\left(\mathbf{k}_{\|} ; \epsilon\right)$ refers to the corresponding similarity transformed $t$-matrix $t_{S}^{p 0}(\epsilon)$, as defined in Eq. (83). The SBZ-integral, Eq. (82), can therefore be expressed as

$$
\tau^{p q}(\epsilon)=\sum_{S \in G} D(S)^{+}\left[\frac{1}{\Omega_{\mathrm{IBZ}_{1}}} \int_{\mathrm{IBZ}_{1}} \tau_{S}^{p q}\left(\mathbf{k}_{\|} ; \epsilon\right) \mathrm{d} \mathbf{k}_{\|}\right] D(S),
$$

where $\Omega_{\mathrm{IBZ}}$ denotes the surface area of $\mathrm{IBZ}_{1}$. Eq. (88) implies (i) that the structure constants need only be evaluated for a chosen set of $\mathbf{k}_{\|} \in \mathrm{IBZ}_{1}$ and (ii) that for any pair $S, R \in G$ for which $t_{S}^{p 0}(\epsilon)=t_{R}^{p 0}(\epsilon)$ the integrals in Eq. (88) are identical.

\subsection{Physical observables}

Based on the Green function in Eq. (56) physical observables can be evaluated in the usual manner, see, e.g., [35]. For matters of completeness below, only the corresponding expressions for the spin-only magnetic moments $m_{\text {spin }}$ and the orbital magnetic moments $m_{\mathrm{orb}}$ (in units of $\mu_{B}$ ) are given,

$m_{\text {spin }}=-\frac{1}{\pi} \operatorname{Im} \int_{\mathscr{C}} \operatorname{Tr}\left\{\beta \sigma_{z} G(z)\right\} \mathrm{d} z$,

$m_{\mathrm{orb}}=-\frac{1}{\pi} \operatorname{Im} \int_{\mathscr{C}} \operatorname{Tr}\left\{\beta L_{z} G(z)\right\} \mathrm{d} z$,

where $\mathscr{C}$ denotes an integration contour in the upper half of the complex energy plane, which starts at the real axis below the valence band ("band bottom") and ends at the Fermi energy, Tr denotes the trace in the tensorial space of spin and configuration, and $L_{z}$ is the $z$ component of the angular momentum operator.

\subsection{Atomic sphere approximation}

Frequently in practical calculations the socalled atomic sphere approximation (ASA) is used in which the unit volume $\Omega^{p}$ corresponding to the origin of the two-dimensional lattice characterizing atomic layer $p$ is replaced by a sphere of equal volume,

$\frac{4 \pi}{3}\left(R_{\mathrm{WS}}^{p}\right)^{3}=\Omega^{p}$.

The radius of this sphere - the Wigner-Seitz radius - is then used to (a) calculate respective single site 
$t$-matrices and (b) determine the normalization integrals of scattering solutions needed to evaluate densities of states, Bloch spectral functions, or magnetic moments, see in particular the discussion in [35], i.e., $R_{\mathrm{WS}}^{p}$ replaces uniformly the muffin-tin radii.

\section{The coherent potential approximation for layered systems}

For a given intermediate region of $n$ layers the so-called coherent scattering path operator $\tau_{c}(z)$ is defined by the following SBZ integral [5],

$$
\begin{aligned}
\tau_{c}^{p i, q j}(z)= & \Omega_{\mathrm{SBZ}}^{-1} \int \exp \left[-\mathrm{i} \mathbf{k}_{\|} \cdot\left(\mathbf{R}_{i, \|}-\mathbf{R}_{j, \|}\right)\right] \\
& \times \tau_{c}^{p q}\left(\mathbf{k}_{\|}, z\right) \mathrm{d} \mathbf{k}_{\|},
\end{aligned}
$$

which implies that in each layer $p$ for the coherent single-site t-matrices the following translational invariance applies:

$t_{c}^{p i}(z)=t_{c}^{p}(z) \quad \forall i \in I(\mathscr{L})$.

In Eq. (91) it is supposed that in all atomic layers one and the same two-dimensional translational symmetry applies, i.e., $\mathscr{L}\left(R_{i, z}\right)=\mathscr{L} \forall R_{i, z}$, see also Eq. (28), where $\mathscr{L}$ refers to a simple two-dimensional lattice, and - as in the previous section position vectors are simply denoted by $\mathbf{R}_{p i}$

$$
\mathbf{R}_{p i}=\mathbf{C}_{p}+\mathbf{R}_{i, \|}, \quad \mathbf{R}_{i, \|} \in \mathscr{L}, \mathbf{C}_{p}=R_{i, z} \mathbf{z} .
$$

In Eq. (91) and in the following (super-) matrices, labelled by layers only shall be used:

$\mathbf{t}_{c}(z)=\left(\begin{array}{ccccc}t_{c}^{11}(z) & 0 & \cdots & & 0 \\ & \ddots & & & \\ 0 & \cdots & t_{c}^{p p}(z) & \cdots & 0 \\ & & & \ddots & \\ 0 & & \cdots & 0 & t_{c}^{n n}(z)\end{array}\right)$,

$$
\tau_{c}(z)=\left(\begin{array}{ccccc} 
& \vdots & & \vdots & \\
\cdots & \tau_{c}^{p p}(z) & \cdots & \tau_{c}^{p q}(z) & \cdots \\
& \vdots & & \vdots & \\
\cdots & \tau_{c}^{q p}(z) & \cdots & \tau_{c}^{q q}(z) & \cdots \\
& \vdots & & \vdots &
\end{array}\right) \text {, }
$$

with $t_{c}^{p p}(z) \equiv t_{c}^{p}(z)$ and $p, q=1, \ldots, n$. Quite clearly a particular element of $\tau_{c}(z)$,

$$
\tau_{c}^{p q}(z)=\tau_{c}^{p i, q i}(z)=\tau_{c}^{p 0, q 0}(z)=\Omega_{\mathrm{SBZ}}^{-1} \int \tau_{c}^{p q}\left(\mathbf{k}_{\|}, z\right) \mathrm{d} \mathbf{k}_{\|},
$$

refers to the unit cells $(i=0)$ at the origin of $\mathscr{L}$ in layers $p$ and $q$. Suppose now the concentration for constituents $A$ and $B$ in layer $p$ is denoted by $c_{p}^{\alpha}$ $(p=1, \ldots, n)$, and one specifies the occupation in the unit cell at the origin of $\mathscr{L}$ of a particular layer $p$ in terms of the following matrix $\mathbf{m}_{p \alpha}(z)$

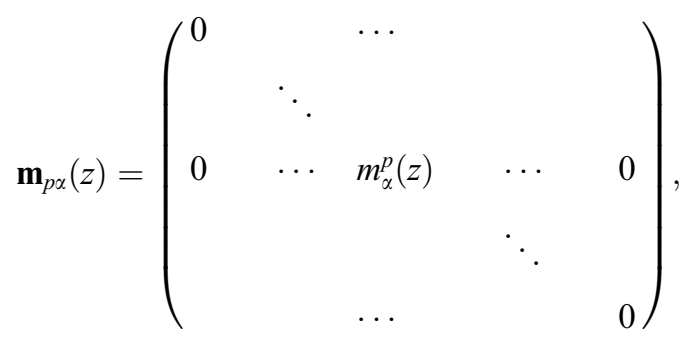

$m_{\alpha}^{p}(z)=t_{c}^{p}(z)^{-1}-t_{\alpha}^{p}(z)^{-1}, \quad \alpha=A, B$,

where $t_{\alpha}^{p}(z)$ is the single-site t-matrix for constituent $\alpha$ in layer $p$. The corresponding layer-diagonal element of the so-called impurity matrix is then given by

$D_{\alpha}^{p p}(z) \equiv D_{\alpha}^{p 0, p 0}(z)=\left[1-m_{\alpha}^{p}(z) \tau_{c}^{p 0, p 0}(z)\right]^{-1}$,

and specifies a single impurity of type $\alpha$ in the translational invariant "host" formed by layer $p$. The coherent scattering path operator for the intermediate region (multilayer) $\tau_{c}(z)$ is therefore obtained from the following inhomogeneous coherent potential approximation (CPA) condition [13], 
$\tau_{c}^{p p}(z)=\sum_{\alpha=A, B} c_{p}^{\alpha}\left\langle\tau^{p p}(z)\right\rangle_{p, \alpha}$,

$\left\langle\tau^{p p}(z)\right\rangle_{p, \alpha}=\tau_{\alpha}^{p p}(z)=D_{\alpha}^{p p}(z) \tau_{c}^{p p}(z), \quad p=1, \ldots, n$,

i.e., from a condition that implies solving simultaneously a layer-diagonal CPA condition for layers $p=1, \ldots, n$. Once this condition is met then translational invariance in each layer under consideration is achieved,

$$
\begin{gathered}
\left\langle\tau^{p p}(z)\right\rangle_{p, \alpha} \equiv\left\langle\tau^{p 0, p 0}(z)\right\rangle_{p 0, \alpha}=\left\langle\tau^{p i, p i}(z)\right\rangle_{p i, \alpha} \\
\forall i \in I(\mathscr{L}), \quad \alpha=A, B, \quad p=1, \ldots, n .
\end{gathered}
$$

Similarly, by specifying the occupation in two different sites, see e.g. [35], the following restricted averages are obtained,

$$
\begin{aligned}
p & \neq q:\left\langle\tau^{p i, q j}(z)\right\rangle_{p i \alpha, q j \beta} \\
& =D_{\alpha}^{p p}(z) \tau_{c}^{p i, q j}(z) D_{\beta}^{q q}(z)^{t} \quad \forall i, j \in I(\mathscr{L}), \\
p & =q:\left\langle\tau^{p i, p j}(z)\right\rangle_{p i \alpha, p j \beta} \\
& =D_{\alpha}^{p p}(z) \tau_{c}^{p i, p j}(z) D_{\beta}^{p p}(z)^{t} \quad \forall(i \neq j) \in I(\mathscr{L}),
\end{aligned}
$$

where $\left\langle\tau_{c}^{p i, q j}(z)\right\rangle_{p i \alpha, q j \beta}$ has the meaning that site (subcell) $p i$ is occupied by species $\alpha$ and site (subcell) $q j$ by species $\beta$ and the symbol $t$ indicates a transposed matrix.

\section{Anisotropy energies}

\subsection{The band energy contribution}

In general, as already stated previously, a magnetic anisotropy energy is defined as the energy difference between two different magnetic configurations. Using a Kohn-Sham-Dirac Hamiltonian as given in Eq. (14) such an anisotropy energy has to be compiled from the respective total energy difference and a contribution reflecting the energy difference arising from the different magnetic dipole-dipole interactions. This term, which is not included in a density functional-like approach is usually described classically.

Restricting in the following magnetic anisotropies to the special case of perpendicular anisotro- pies, the magnetic anisotropy energy $\Delta E_{a}(N)$ is then defined as the energy difference between a uniform in-plane $(\delta=\|)$ and a uniform normal-toplane $(\delta=\perp)$ orientation of the magnetization,

$\Delta E_{a}(N)=\Delta E(N)+\Delta E_{d d}(N)$,

where $\Delta E(N)$ and $\Delta E_{d d}(N)$ are the corresponding total energy and magnetostatic dipole-dipole energy difference, respectively,

$\Delta E(N)=E(N ; \|)-E(N ; \perp)$,

$\Delta E_{d d}(N)=E_{d d}(N ; \|)-E_{d d}(N ; \perp)$,

and $N$ specifies the number of atomic layers in the system of consideration. Relying on the "Magnetic Force Theorem" (see e.g. Ref. [42]) $\Delta E(N)$ can be replaced by the corresponding difference $\Delta E_{b}(N)$ of the grand potentials $E_{b}(N ; \delta),(\delta=\|$ or $\perp)$, see also the following section.

\subsubsection{Layer-resolved band energy contributions}

In the more general case of (statistically) disordered multilayers, i.e., when using the CPA, $E_{b}(N ; \delta)$ is defined as

$$
\begin{aligned}
& E_{b}(N ; \delta)=\sum_{p=1}^{N} \sum_{\alpha} c_{p}^{\alpha} E_{b}^{p, \alpha}(\delta), \\
& E_{b}^{p, \alpha}(\delta)=\int_{-\infty}^{\epsilon_{\mathrm{F}}}\left(\epsilon-\epsilon_{\mathrm{F}}\right) n_{p}^{\alpha}(\epsilon ; \delta) \mathrm{d} \epsilon,
\end{aligned}
$$

where $p$ specifies a certain layer, $\alpha$ refers to a particular component of concentration $c_{p}^{\alpha}$ and the $n_{p}^{\alpha}(\epsilon ; \delta)$ are the layer and component projected local densities of states (see also Ref. [13]). Since

$\epsilon_{\mathrm{F}} \int_{-\infty}^{\epsilon_{F}}\left(n_{p}^{\alpha}(\epsilon ; \|)-n_{p}^{\alpha}(\epsilon ; \perp)\right) \mathrm{d} \epsilon$

is rather very small, $\Delta E_{b}(N)$ is usually termed "band energy contribution". It should be noted that in the case of a non-magnetic substrate, one and the same Fermi level $\epsilon_{\mathrm{F}}$ applies for all magnetic configurations.

\subsubsection{Discrete Fourier transformations}

Consider the following "capped trilayer system", where (hkl) is the surface orientation of the substrate and the cap can consists of $r$ layers of a 
suitable metal (not necessarily the same as the substrate),

Substrate $(\mathrm{hkl}) / \mathrm{Co}_{n} \mathrm{Cu}_{m} \mathrm{Co}_{n} /(\mathrm{Cap})_{r}$.

Possible oscillatory behavior of $\Delta E_{b}(n, m ; r)$ with respect to either the number of Co layers $n$, the number of $\mathrm{Cu}$-spacer layers $m$, or the number of cap layers $r$, can be detected best in terms of discrete (linear) Fourier transforms. By keeping, for example, $m$ and $r$ constant such a Fourier transformation with respect to a varying number of $\mathrm{Co}$ layers, $n=1, \ldots, N$, is defined by

$F(q ; m ; r)=\frac{1}{N} \sum_{n=1}^{N} \omega(n) \exp (\mathrm{i} q n) \Delta E_{b}(n, m ; r)$,

where usually a prefactor $\omega(n)=n^{2}$ is applied. The positions $q_{i}$ of pronounced maxima of $|F(q ; m ; r)|$ then describe the periods of the oscillations. Furthermore, a discrete Fourier transform of $\Delta E_{b}(n, m ; r)$ with respect to a reference level can be defined,

$\Delta F(q ; m ; r)=\frac{1}{N} \sum_{n=1}^{N} \omega(n) \exp (\mathrm{i} q n)\left(\Delta E_{b}(n, m ; r)-E_{0}\right)$,

where $E_{0}$ is given, e.g., by either the following mean value

$E_{0}=\frac{1}{N} \sum_{n=1}^{N} \Delta E_{b}(n, m ; r)$

or by a so-called biased value such as $E_{a}(n, \infty)$, where taking the example in (107) $E_{a}(n, \infty)$ corresponds to $\mathrm{Co}_{n}$ multilayers on $\mathrm{Cu}(100)$ with a semi-infinite $\mathrm{Cu}$ cap.

In the same manner layer-resolved quantities such as layer-resolved magnetic moments or band energy differences can be discrete Fourier transformed. In such a case the summation is to be taken over all layers in a particular system,

$F(q ; m ; r)=\frac{1}{P} \sum_{p=1}^{P} \exp (\mathrm{i} q p) \Delta E_{b}^{p, \alpha}(n, m ; r)$,

such as $P=2 n+m+r$ for the example given in (107).

\subsubsection{Intrinsic quantities}

Suppose the magnetic anisotropy energy or other physical properties of repeated magnetic multilayer systems such as superstructures of Co and Pd on a Pd substrate

$\mathrm{Pd}(\mathrm{hkl}) /\left(\mathrm{Co}_{n} \mathrm{Pd}_{m}\right)_{r}$

have to be described theoretically. Use of Eq. (111) with $P=r \times(n+m)$ will then show the periods of repetition for a particular physical property $O(r)$. Clearly enough these periods can be multiples of $n, m$ or $(n+m)$. In order to call such a system "colloquially" a superlattice, in addition to welldeveloped peaks in $|F(q ; m ; r)|$ at $q_{0}=L r, L$ being an integer number, a characteristic volume also has to be defined. If therefore for a sufficiently large $R$,

$\lim _{r \rightarrow R} \frac{\mathrm{O}(r)}{r}=\mathrm{O}(R) \sim \overline{\mathrm{O}}=$ const,

i.e., if $\mathrm{O}(R)$ depends only very weakly on the number of repetitions, then $\mathrm{O}(R) / R$ can be regarded as a kind of "intrinsic" quantity or "saturation value".

\subsection{Magnetic dipole-dipole energy contribution}

\subsubsection{The classical magnetic dipole-dipole interac- tion}

If one partitions the configurational space into cells centered around positions $\mathbf{R}$, then within the dipole approximation the relativistic current-current interaction energy is reduced to the magnetostatic dipole-dipole interaction energy, which can be expressed [37] (in atomic rydberg units) as

$$
\begin{aligned}
E_{d d}= & \frac{1}{c^{2}} \sum_{\mathbf{R}, \mathbf{R}^{\prime}}^{\prime}\left\{\frac{\mathbf{m}_{\mathbf{R}} \mathbf{m}_{\mathbf{R}^{\prime}}}{\left|\mathbf{R}-\mathbf{R}^{\prime}\right|^{3}}\right. \\
& \left.-3 \frac{\left[\mathbf{m}_{\mathbf{R}} \cdot\left(\mathbf{R}-\mathbf{R}^{\prime}\right)\right]\left[\mathbf{m}_{\mathbf{R}^{\prime}} \cdot\left(\mathbf{R}-\mathbf{R}^{\prime}\right)\right]}{\left|\mathbf{R}-\mathbf{R}^{\prime}\right|^{5}}\right\},
\end{aligned}
$$

where $\mathbf{m}_{\mathbf{R}}$ is the magnetic moment at site $\mathbf{R}=\mathbf{R}_{p \alpha}+\mathbf{R}_{\|}, \mathbf{R}_{\|} \in \mathscr{L}$, with $\mathbf{R}_{p \alpha}$ specifying a site in the unit cell of the system at layer $p$ and sublattice $\alpha$. The primed sum indicates a restriction to $\mathbf{R} \neq \mathbf{R}^{\prime}$. Since two-dimensional translational 
invariance pertains within the layers, Eq. (114) can be rewritten as

$E_{d d}=\sum_{p \alpha, q \beta} \frac{m_{p \alpha} m_{q \beta}}{c^{2}} \widehat{\mathbf{n}} \underline{M}_{p \alpha, q \beta}^{d d} \widehat{\mathbf{n}}$,

$6 \mathrm{ptwhere} \widehat{\mathbf{n}}$ refers to a given uniform orientation of the magnetization (magnetic moments). In (115) the so-called two-dimensional (ferromagnetic) dipole-dipole Madelung constant matrix is defined by

$$
\begin{aligned}
& \underline{M}_{p \alpha, q \beta}^{d d}=\sum_{\mathbf{R}_{\|}}^{\prime} \frac{1}{\left|\mathbf{R}_{p \alpha}-\mathbf{R}_{q \beta}-\mathbf{R}_{\|}\right|^{3}} \\
& \quad \times\left\{\underline{I}-\frac{\left(\mathbf{R}_{p \alpha}-\mathbf{R}_{q \beta}-\mathbf{R}_{\|}\right) \otimes\left(\mathbf{R}_{p \alpha}-\mathbf{R}_{q \beta}-\mathbf{R}_{\|}\right)}{\left|\mathbf{R}_{p \alpha}-\mathbf{R}_{q \beta}-\mathbf{R}_{\|}\right|^{2}}\right\},
\end{aligned}
$$

where $I$ is a $3 \times 3$ unit matrix and $\otimes$ denotes a tensorial product of vectors. In (116) the prime

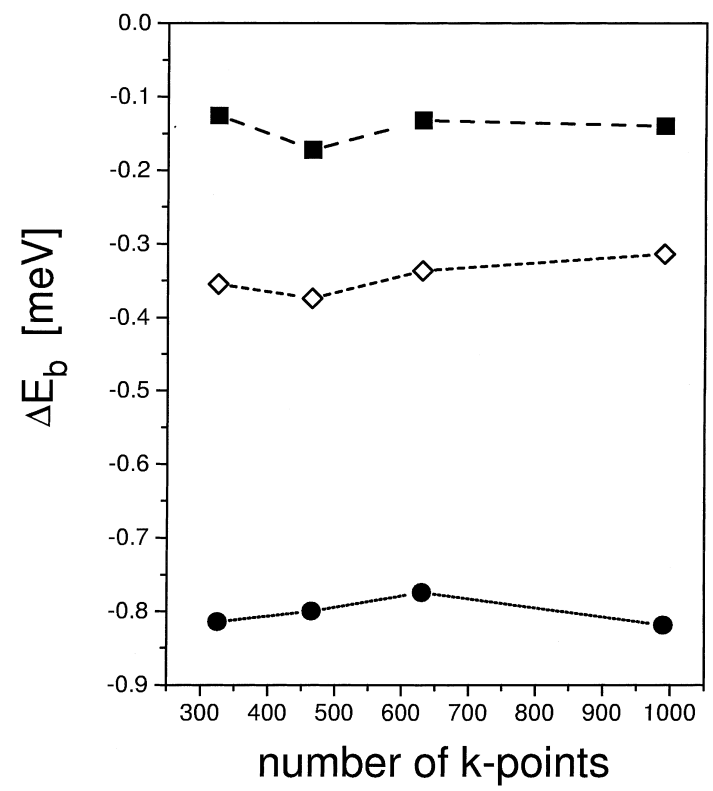

Fig. 1. Convergence of the band energy contribution to the magnetic anisotropy energy with respect to the number of k-points used in the irreducible part of the SBZ. Diamonds: free surface of $\mathrm{Cu}(100) / \mathrm{Co}_{11}$; squares: $\mathrm{Cu}(100) / \mathrm{CuCo}_{11} \mathrm{Cu} /$ $\mathrm{Cu}\left(\begin{array}{lll}1 & 0 & 0\end{array}\right)$, and circles: $\mathrm{Cu}\left(\begin{array}{lll}1 & 0 & 0\end{array}\right) / \mathrm{AuCo}_{11} \mathrm{Au} / \mathrm{Cu}\left(\begin{array}{lll}1 & 0 & 0\end{array}\right)$. indicates that for $\mathbf{R}_{p \alpha}=\mathbf{R}_{q \beta}$ the singular term corresponding to $\mathbf{R}_{\|}=0$ is excluded from the summation.

For $\left|R_{p \alpha ; \perp}-R_{q \beta ; \perp}\right| \neq 0$ the magnetostatic Poisson equation can be solved in a straightforward manner (see also Ref. [38]) by using a Fourier expansion [39] that results into a fast convergent series

$$
\begin{aligned}
& \underline{M}_{p \alpha, q \beta}^{d d}=-\frac{2 \pi}{A} \sum_{G_{\|} \neq 0}\left|\mathbf{G}_{\|}\right| \exp \left(-\left|\mathbf{G}_{\|}\right|\left|R_{p \alpha ; \perp}-R_{q \beta ; \perp}\right|\right) \\
& \times \exp \left(\mathbf{i} \mathbf{G}_{\|} \cdot\left(\mathbf{R}_{p \alpha ; \|}-\mathbf{R}_{q \beta ; \|}\right)\right) \\
& \times\left(\begin{array}{cc}
-\frac{\mathbf{G}_{\|} \otimes \mathbf{G}_{\|}}{\left|\mathbf{G}_{\|}\right|^{2}} & -\operatorname{isgn}\left(R_{p \alpha ; \perp}-R_{q \beta ; \perp}\right) \frac{\mathbf{G}_{\|} \otimes 1}{\left|\mathbf{G}_{\|}\right|} \\
-\operatorname{isgn}\left(R_{p x ; \perp}-R_{q \beta ; \perp}\right) \frac{1 \otimes \mathbf{G}_{\|}}{\left|\mathbf{G}_{\|}\right|} & 1
\end{array}\right),
\end{aligned}
$$

where $A$ is the two-dimensional unit cell area and the $\mathbf{G}_{\|}$are two-dimensional reciprocal space vectors. It should be noted that the $\mathbf{G}_{\|}=0$ component does not contribute to the 'off-plane' terms. For the (lllll) and (1 $\left.\begin{array}{lll}1 & 1 & 1\end{array}\right)$ faces of the simple cubic

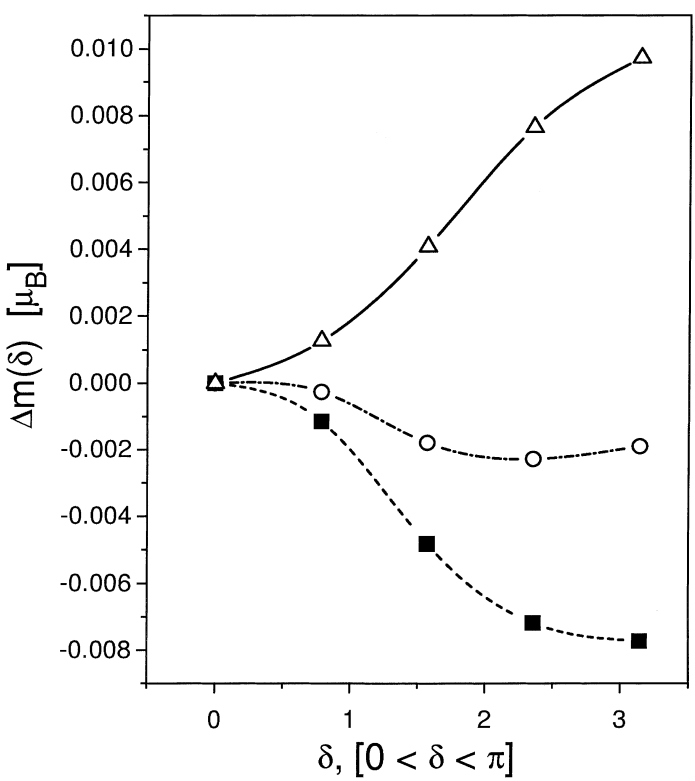

Fig. 2. Layer-resolved magnetic moment differences with respect to the ferromagnetic alignment in $\mathrm{Au}(100) / \mathrm{Fe} \mathrm{Au}_{3} \mathrm{Fe} /$

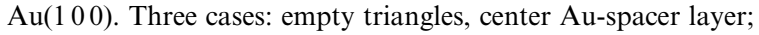
filled squares, Au-spacer layers at the $\mathrm{Fe} / \mathrm{Au}$ interface; empty circles, Fe-layers. See also Ref. [19]. 
lattices (sc, bcc or fcc) Eq. (117) can be further reduced to

$\underline{M}_{p q}^{d d}=M_{p q}^{d d} \times\left(\begin{array}{rrr}-\frac{1}{2} & 0 & 0 \\ 0 & -\frac{1}{2} & 0 \\ 0 & 0 & 1\end{array}\right)$,

where

$$
\begin{aligned}
M_{p q}^{d d}= & -\frac{2 \pi}{A} \sum_{G_{\| \neq 0}}\left|\mathbf{G}_{\|}\right| \exp \left(-\left|\mathbf{G}_{\|}\right|\left|R_{p ; \perp}-R_{q ; \perp}\right|\right) \\
& \times \cos \left(\mathbf{G}_{\|} \cdot\left(\mathbf{R}_{p ; \|}-\mathbf{R}_{q ; \|}\right)\right) .
\end{aligned}
$$

For this special case the two-dimensional ferromagnetic dipole-dipole Madelung constants for $p=q$ can be evaluated by using a standard Ewald summation technique,

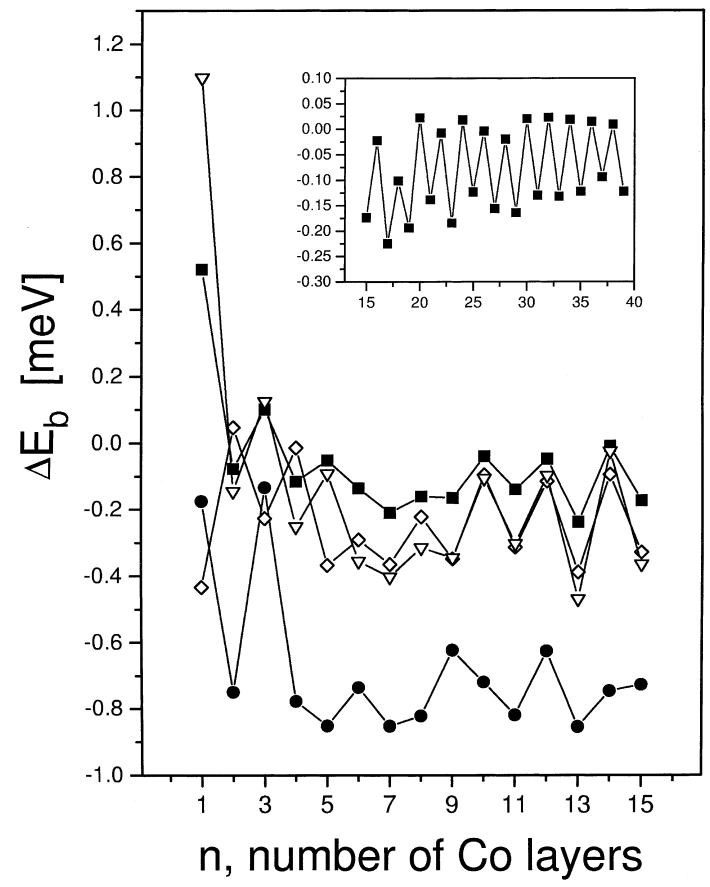

Fig. 3. Band energy contribution to the magnetic anisotropy energy as a function of Co layers. Diamonds: free surfaces of $\mathrm{Cu}(100) / \mathrm{Co}_{n}$; squares: $\mathrm{Cu}(100) / \mathrm{CuCo}_{n} \mathrm{Cu} / \mathrm{Cu}(100)$; circles: $\mathrm{Cu}\left(\begin{array}{lll}1 & 0 & 0\end{array}\right) / \mathrm{AuCo} \mathrm{C}_{n} \mathrm{Au} / \mathrm{Cu}\left(\begin{array}{lll}1 & 0 & 0\end{array}\right)$, and up-triangles: $\mathrm{Cu}\left(\begin{array}{lll}1 & 0 & 0\end{array}\right) /$ $\mathrm{Co}_{n} \mathrm{Cu}_{m} \mathrm{Co}_{n} / \mathrm{Cu}(100)$. The inset shows the continuation for $n$ $\geqslant 15$ for the $\mathrm{Cu}(100) / \mathrm{Co}_{n} / \mathrm{Cu}(100)$ systems. From Ref. [20].

$$
\begin{aligned}
M_{p p}^{d d}= & \sum_{\mathbf{R}_{\| \neq 0}}\left\{\frac{\operatorname{erf}_{\mathrm{c}}\left(\left|\mathbf{R}_{\|}\right| / 2 \sigma\right)}{\left|\mathbf{R}_{\|}\right|^{3}}+\frac{\exp \left(-\left|\mathbf{R}_{\|}\right|^{2} / 4 \sigma^{2}\right)}{\sigma \sqrt{\pi}\left|\mathbf{R}_{\|}\right|^{2}}\right\} \\
& -\frac{2 \pi}{A} \sum_{\mathbf{G}_{\| \neq 0}}\left\{\left|\mathbf{G}_{\|}\right| \operatorname{erf}_{\mathrm{c}}\left(\left|\mathbf{G}_{\|}\right| \sigma\right)\right. \\
& \left.-\frac{1}{\sigma \sqrt{\pi}} \exp \left(-\left|\mathbf{G}_{\|}\right|^{2} \sigma^{2}\right)\right\} \\
& -\frac{1}{6 \sigma^{3} \sqrt{\pi}}+\frac{2 \sqrt{\pi}}{A \sigma},
\end{aligned}
$$

where $\sigma$ is the Ewald parameter and $\operatorname{erf}_{\mathrm{c}}(x)=$ $1-\operatorname{erf}(x)$ [40]. Finally, by using polar coordinates for the unit vector $\widehat{\mathbf{n}}=(\sin (\Theta) \cos (\phi), \sin (\Theta)$ $\sin (\phi), \cos (\Theta))$, one immediately obtains that

$\widehat{\mathbf{n}}\left(\begin{array}{rrr}-\frac{1}{2} & 0 & 0 \\ 0 & -\frac{1}{2} & 0 \\ 0 & 0 & 1\end{array}\right) \widehat{\mathbf{n}}=\frac{3}{2} \cos ^{2}(\Theta)-\frac{1}{2}$,

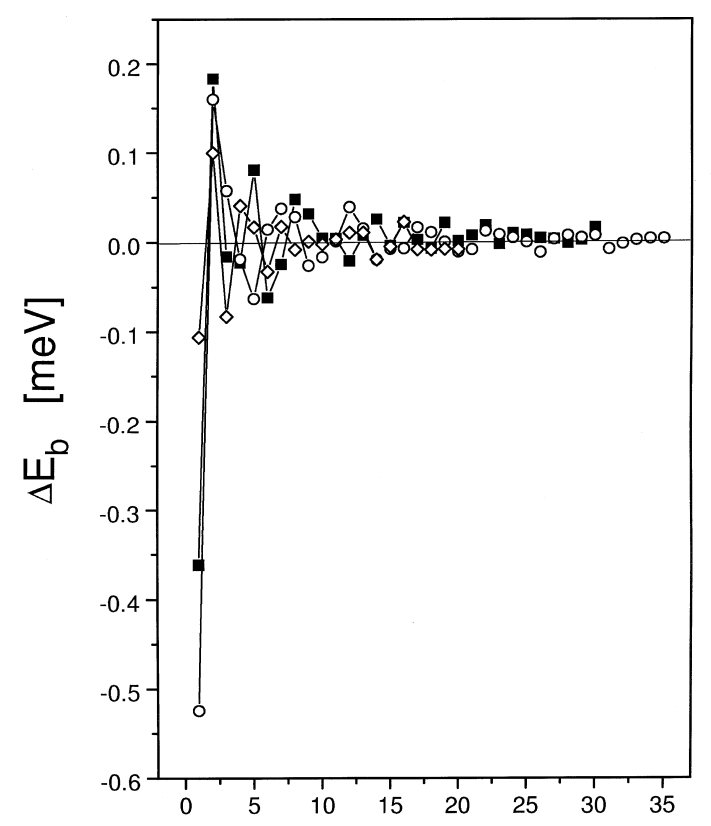

$\mathrm{m}$, number of Cu-spacer layers

Fig. 4. Relative band energy contribution to the magnetic anisotropy energy as a function of Cu-spacer layers $m$ in $\mathrm{Cu}(100) /$ $\mathrm{Co}_{n} \mathrm{Cu}_{m} \mathrm{Co}_{n} / \mathrm{Cu}(100), n=1$ (circles), 4 (squares), 6 (diamonds). From Ref. [20]. 
which implies that the orientational dependence of the magnetostatic dipole-dipole anisotropy energy, $\Delta E_{d d}$, can be simply written as [38]

$$
\Delta E_{d d}(\Theta)=\left(E_{d d}^{\|}-E_{d d}^{\perp}\right) \sin ^{2}(\Theta) .
$$

\subsubsection{Disordered systems}

In terms of a general inhomogeneous CPA for layerwise, binary substitutionally disordered systems the magnetic moments arising from the constituents have to be weighted with their respective concentrations such that to each site in a given layer $p$ a uniform magnetic moment applies.

$$
\left\langle\mathbf{m}_{p}\right\rangle=\sum_{\alpha} c_{p}^{\alpha} \mathbf{m}_{p}^{\alpha}
$$

where \langle\rangle denotes an average over statistical configurations and $\mathbf{m}_{p}^{\alpha}$ refers to the magnetic moment of component $\alpha$ in layer $p$. It should be noted that by using the above averaged magnetic moments in
Eq. (115), one in fact neglects vertex corrections of the kind $\left\langle\mathbf{m}_{\mathbf{R}} \mathbf{m}_{\mathbf{R}^{\prime}}\right\rangle-\left\langle\mathbf{m}_{\mathbf{R}}\right\rangle\left\langle\mathbf{m}_{\mathbf{R}}^{\prime}\right\rangle$, where $\mathbf{R}$ and $\mathbf{R}^{\prime}$ refer to two different sites.

\section{Applications}

\section{1. $k$-convergence of the band energy contribution}

In order to obtain reliable band energy differences, in principle for every new type of system the convergence of $\Delta E_{b}$ with respect to the number of $\mathbf{k}_{\|}$values used to evaluate the relevant SBZ integrals, see in particular Eq. (88), has to be checked. In Fig. 1 typical examples are shown, namely for a free surface and for sandwich systems. As can be seen from this figure, by using more than $900 \mathbf{k}_{\|}$ values $\in \mathrm{SBZ}_{1}$, usually rather well-converged values can be expected, the inherent error being of the order of $0.01-0.02 \mathrm{MeV}$. It should be noted that in order to achieve self-consistency for the effective
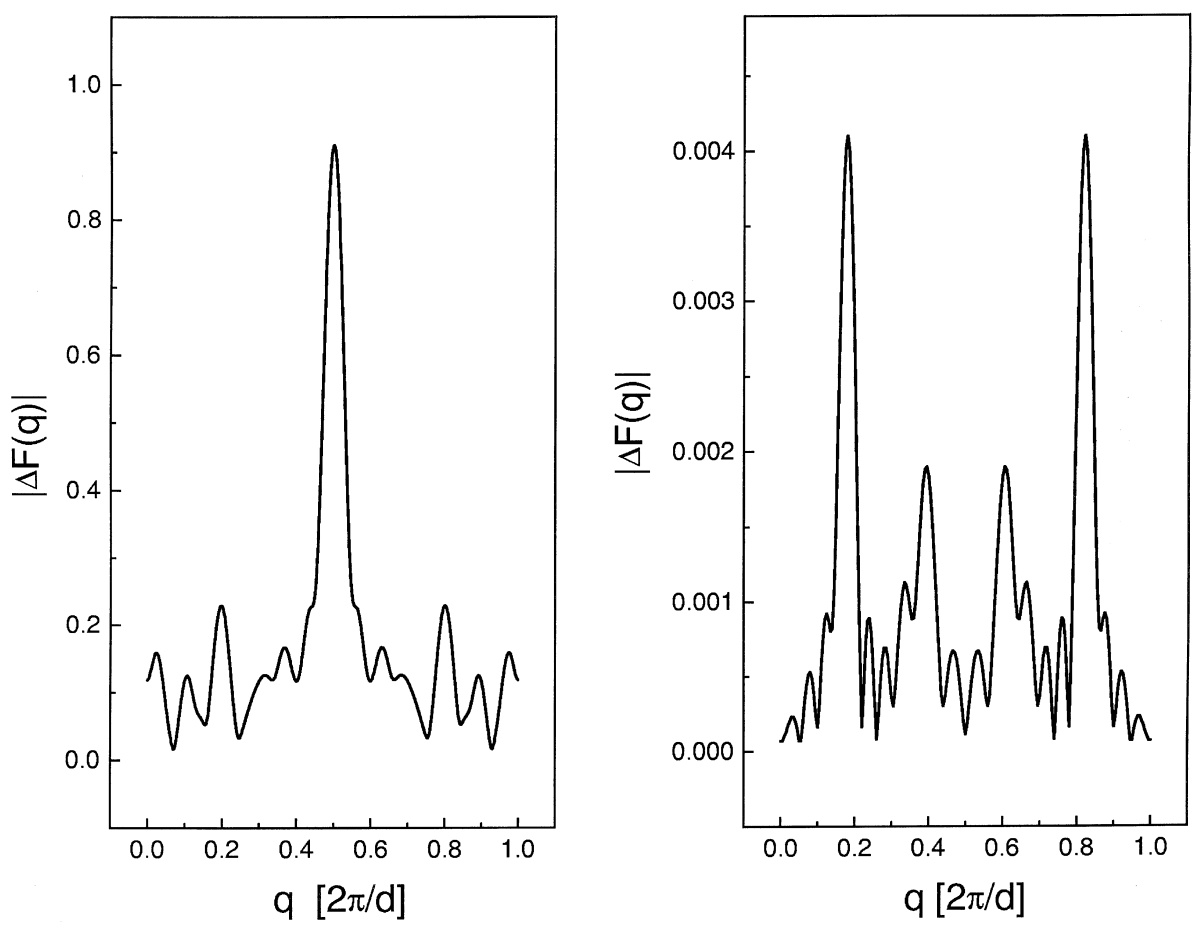

Fig. 5. Absolute values of discrete Fourier transformations as a function of $q$. Left panel: with respect to the number of Co layers $n$ in $\mathrm{Cu}\left(\begin{array}{ll}0 & 0\end{array}\right) / \mathrm{Co}_{n} / \mathrm{Cu}\left(\begin{array}{ll}0 & 0\end{array}\right)$, right panel: with respect to the number of $\mathrm{Cu}$-spacer layers $m$ in $\mathrm{Cu}\left(\begin{array}{ll}0 & 0\end{array}\right) / \mathrm{Co}_{1} \mathrm{Cu}_{m} \mathrm{Co}_{1} / \mathrm{Cu}(100)$ as obtained by excluding the preasymptotic regime. From Ref. [20]. 

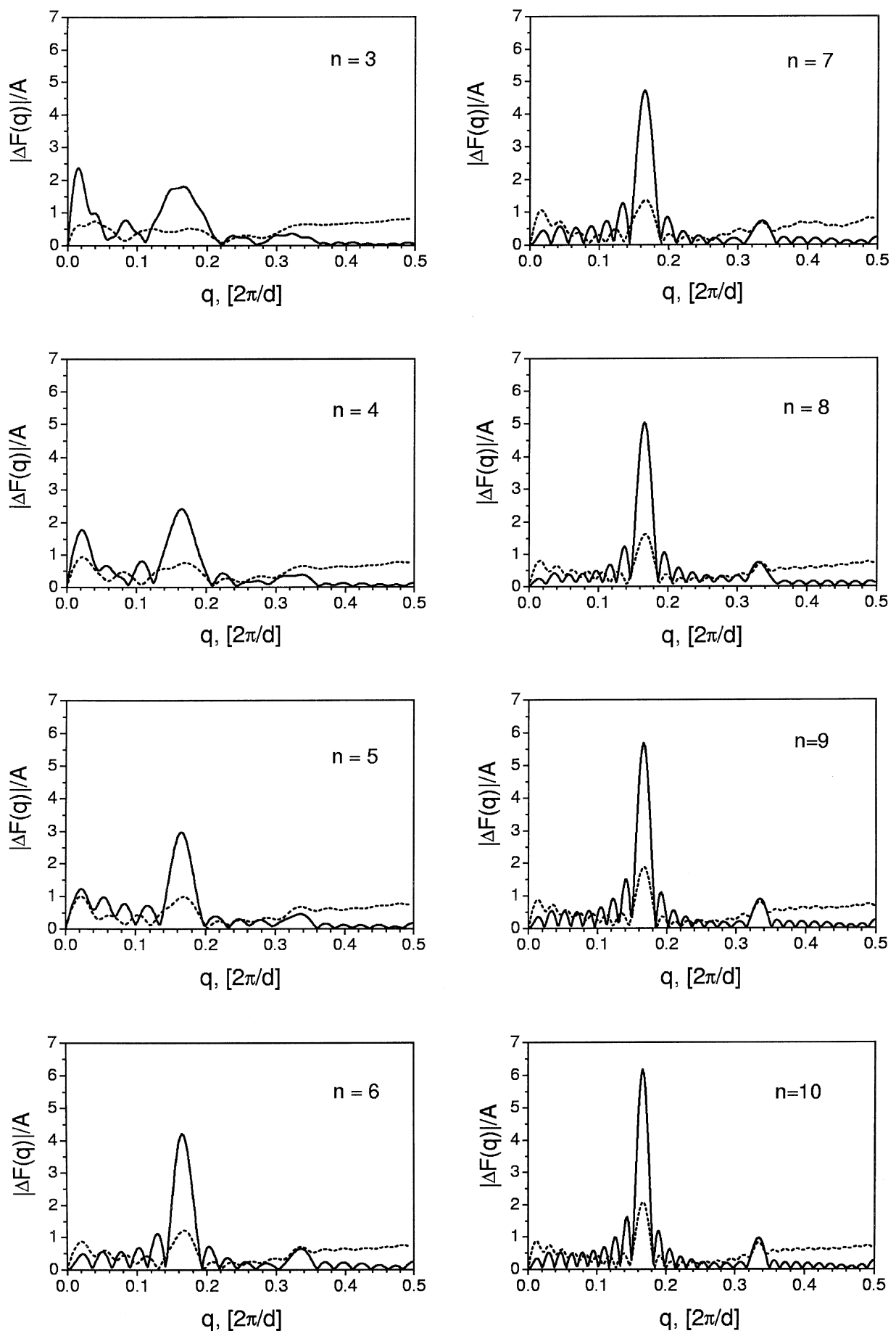

Fig. 6. Absolute values of the discrete Foruier transformation of the layer-resolved band energy contributions to the magnetic anisotropy energy with respect to layers in $\mathrm{Cu}(100) /\left(\mathrm{Cu}_{3} \mathrm{Ni}_{3}\right)_{n}$. The dotted line refers to free surfaces, the full line to a semi-infinite $\mathrm{Cu}$ cap. From Ref. [23]. 
potentials and exchange fields usually substantially less $\mathbf{k}_{\|}$values are needed.

\subsection{Validity of the Magnetic Force Theorem}

By applying the so-called Magnetic Force Theorem, it is assumed that the size of the magnetic moments does not change when changing the orientation of the magnetization either uniformly or in particular layers. Suppose that in the system $\mathrm{Au}(100) / \mathrm{FeAu} \mathrm{H}_{3} \mathrm{Fe} / \mathrm{Au}\left(\begin{array}{lll}1 & 0 & 0\end{array}\right)$ the ferromagnetic configuration corresponds to a uniform in-plane orientation of the magnetization. Suppose further that in one of the Fe layers the orientation of the magnetization is now rotated from parallel to antiparallel, i.e., by an angle $0 \leqslant \delta \leqslant \pi$ around the surface normal. Quite clearly in order to compare total energy differences with differences in band energies based on the Magnetic Force Theorem, both quantities have to be well converged with respect to the number of applied $\mathbf{k}_{\|}$values, see in particular the discussion in [19].

Fig. 2 shows the changes of the (self-consistently obtained) layer-resolved magnetic moments with respect to the ferromagnetic configuration as a function of $\delta$. As can be seen, even in a rather small system, where self-consistency effects got to be rather large, the changes in the magnetic moments are very small. For a rotation by $\pi / 2$ they are of the order of $0.005 \mu_{B}$. Thus in general it can be assumed that the Magnetic Force Theorem applies reasonably well. In most cases it is therefore sufficient to evaluate for a particular system the effective potentials and effective exchange fields self-consistently only for one magnetic configuration (reference configuration) and use the thus obtained effective potentials and effective exchange fields also for other magnetic configurations. This in practical terms is the essence of the Magnetic Force Theorem.

\subsection{Oscillations in the band energy contribution}

Frequently the band energy contribution to the magnetic anisotropy energy shows oscillations either with respect to the number of magnetic layers, the number of spacer layers, the number of cap layers, or with respect to all these parameters.
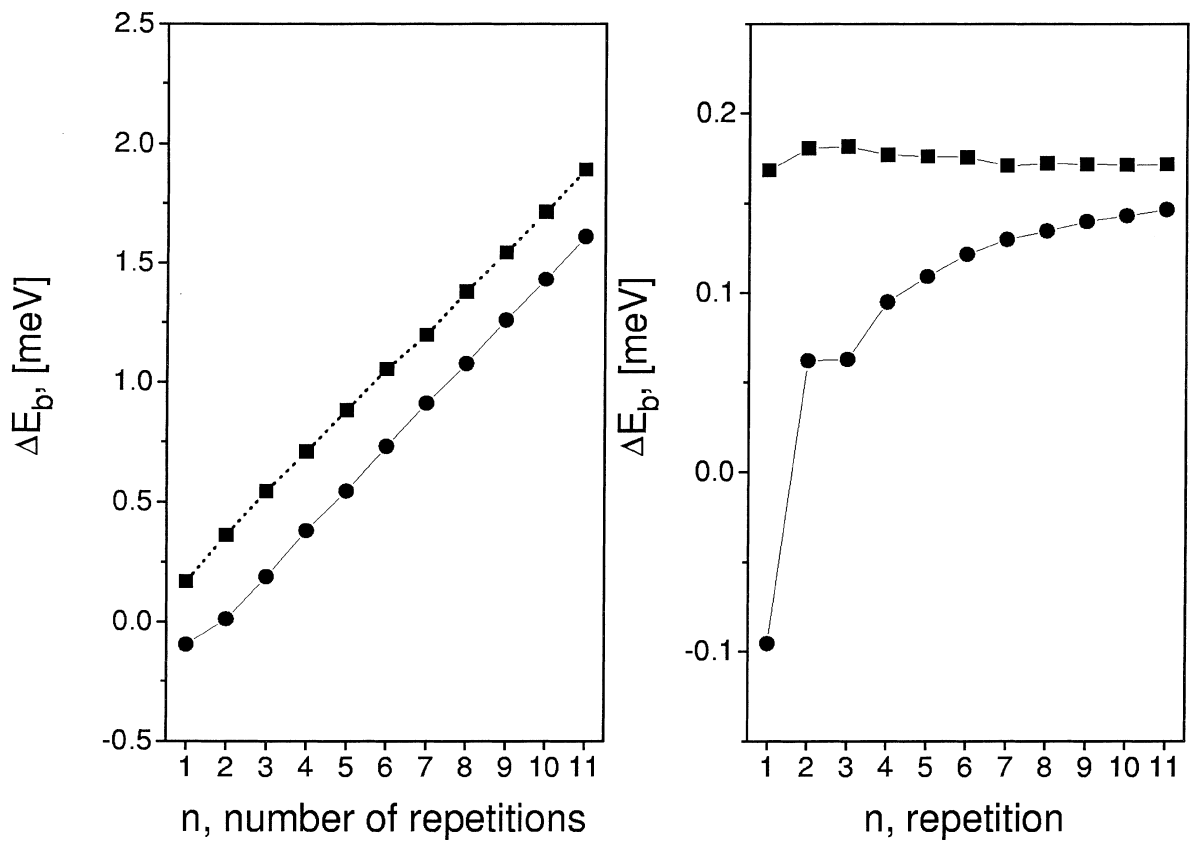

Fig. 7. Band energy contribution to the magnetic anisotropy energy (left) and per repetition (right) in $\mathrm{Cu}(100) /\left(\mathrm{Cu}_{3} \mathrm{Ni}_{3}\right)_{n}$. $\mathrm{Circles}$ refer to free surfaces, squares to a semi-infinite $\mathrm{Cu}$ cap. From Ref. [23]. 
Fig. 3 shows such oscillations for a variety of Corelated multilayer systems on $\mathrm{Cu}(100)$ with respect to the number of Co layers, see in particular [20]. With exception of the system with strongly

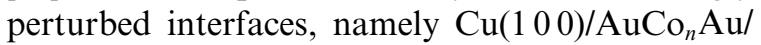

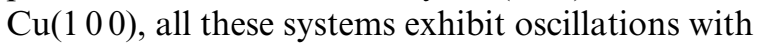
a period of two. Oscillations in $\Delta E_{b}$ with respect to the number of spacer layers in $\mathrm{Cu}(100) /$ $\mathrm{Co}_{n} \mathrm{Cu}_{m} \mathrm{Co}_{n} / \mathrm{Cu}(100)$ are displayed in Fig. 4. Using discrete Fourier transformations, see in particular Eqs. (108) and (109), the periods of such oscillations can easily be traced. As can be seen from Fig. 5, with respect to the number of Co layers there is a peak at $q_{0}=0.5$, the corresponding period, $q_{0}^{-1}$, is exactly what was already read off from Fig. 3. With respect to the number of $\mathrm{Cu}-$ spacer layers two peaks can be traced, the corresponding periods - the so-called short and long period - are exactly those to be seen in an ab initio description of the interface exchange coupling in these systems. This is not really surprising, since the discussion on magnetic configurations made already clear that the so-called interface exchange coupling refers to nothing else but a (band) energy difference between two magnetic configurations.

\subsection{Repetitions, superstructures}

In Fig. 6 discrete Fourier transformations of the layer-resolved $\Delta E_{b}^{n}$ (with respect to the number of layers) are displayed, see Eq. (111), varying the number of repetitions $n$ in the system $\mathrm{Cu}\left(\begin{array}{lll}1 & 0 & 0\end{array}\right) /$ $\left(\mathrm{Cu}_{3} \mathrm{Ni}_{3}\right)_{n}$. As can be seen in this figure, with an increasing number of repetitions, the peak at $q_{0}=1 / 6$ grows in intensity and sharpens up. At least for the band energy part of the anisotropy energy this implies that the thickness (in monolayers) of the repeated unit $\left(\mathrm{Cu}_{3} \mathrm{Ni}_{3}\right)$ is indeed the period of oscillations with respect to the number of layers, see [23]. The other rather small peak at $q_{1}=1 / 3$ is usually referred to as an "aliasing peak". From this figure it is pretty obvious that "periodicity" along the surface normal is much less pronounced for free surfaces than for systems with a semi-infinite $\mathrm{Cu}$-cap.

Fig. 7 shows the band energy contribution $\Delta E_{b}$ and $\Delta E_{b} / n$ with respect to the number of repetitions $n$. As can be seen in there, in the case of free surfaces even after 11 repetitions $\Delta E_{b} / n$ is not really constant, i.e., $\Delta E_{b} / n$ did not become an "intrinsic quantity", while for the capped system, for $n \geqslant 10$ nearly no further changes occur. Quite clearly, at least for free surfaces of this type of system the concept of "superlattices" can hardly be justified.

\subsection{Reorientation transitions}

\subsubsection{The $\mathrm{Fe}_{n} / \mathrm{Au}(100)$ system}

In Fig. 8, a very typical reorientation of the magnetization from perpendicular to in-pane is shown for $\mathrm{Fe}$ multilayers on $\mathrm{Au}(100)$, see [7],

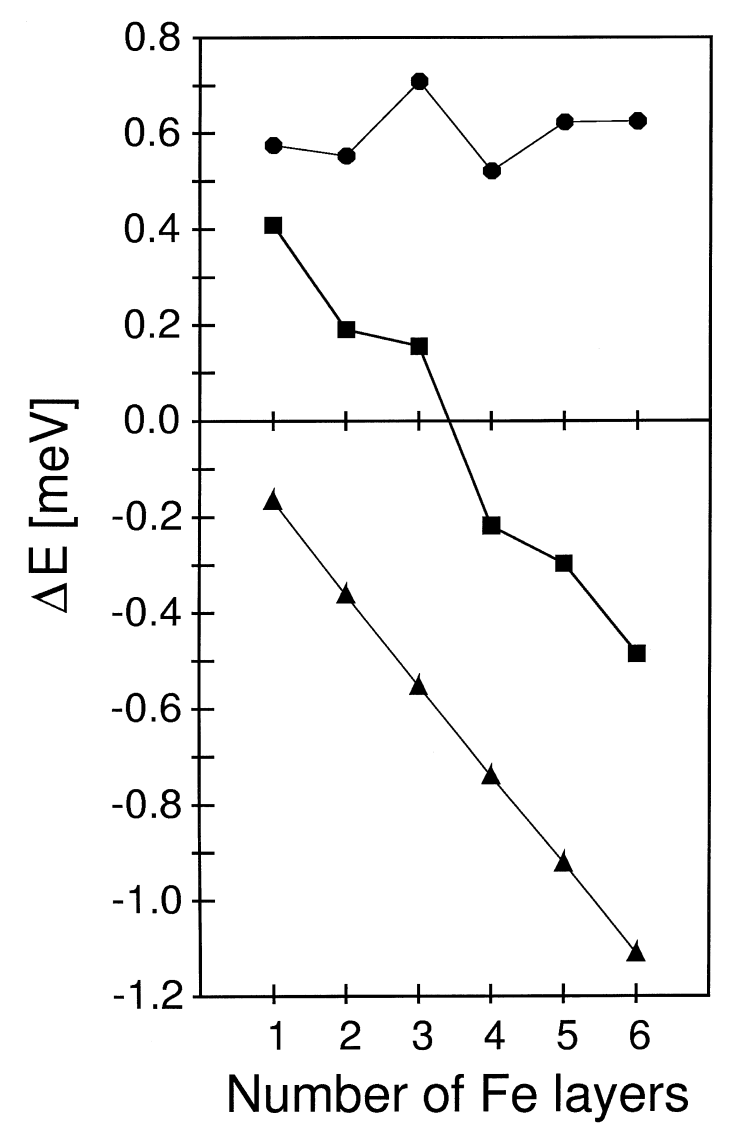

Fig. 8. Reorientation transition for free surfaces of $\mathrm{Fe}$ on

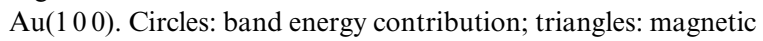
dipole-dipole contribution; squares: magnetic anisotropy energy. From [7]. 
whereby no layer relaxation is assumed with respect to the parent fcc lattice of $\mathrm{Au}$. The reorientation transition occurs between three and four monolayers of $\mathrm{Fe}$, which coincides nicely with available experimental data. Fig. 8 is meant to show in particular the importance of the magnetic dipole-dipole contribution ("shape anisotropy") to the magnetic anisotropy energy, since quite obviously the reorientation transition is driven by this quantity.

\subsection{2. $\mathrm{Fe}_{n} / \mathrm{Cu}(100)$ and $\left(\mathrm{Fe}_{c} \mathrm{Co}_{1-c}\right)_{n} / \mathrm{Cu}(100)$}

According to the literature $\mathrm{Fe}$ overlayers on $\mathrm{Cu}(100)$ seem to be the most disputed system in the field of perpendicular magnetism. Different experimental set-ups and/or growing conditions lead to rather contradicting results. The reasons for all the difficulties arise most likely from the fact that (a) in different experiments different interlayer relaxations pertain, and (b) antiferromagnetic

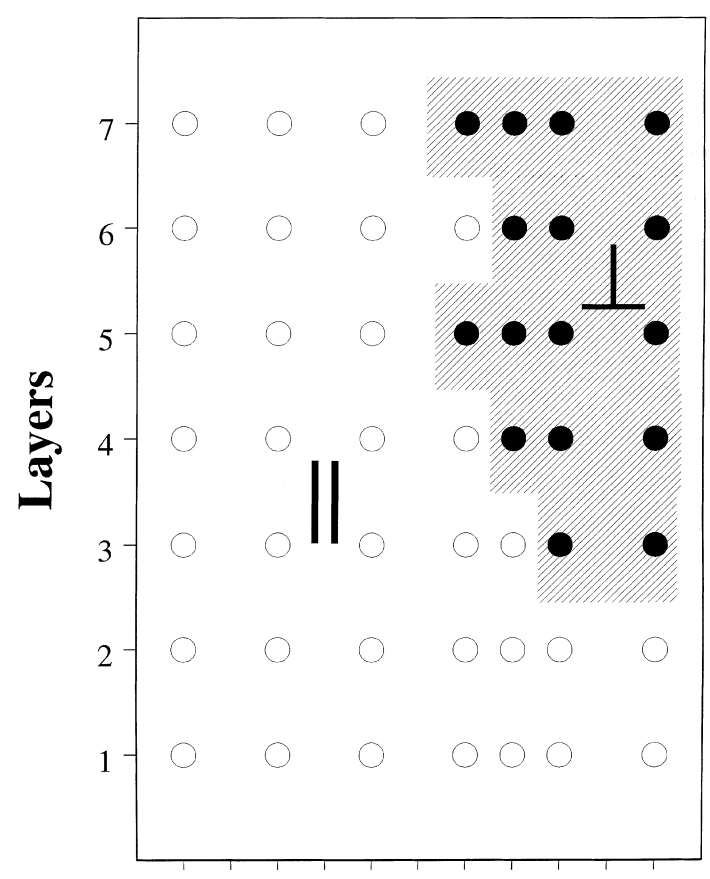

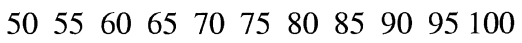

\section{Fe concentration (\%)}

Fig. 9. Phase diagram of the reorientation transition for $\mathrm{Cu}(100) /\left(\mathrm{Fe}_{c} \mathrm{Co}_{1-c}\right)_{n}$. From [24]. coupling between the Fe layers occurs, the type of which can again depend on different interlayer spacings, see also $[12,16,18]$. In Fig. 9 a "phase diagram" of the reorientation transition in $\mathrm{Cu}\left(\begin{array}{ll}1 & 0\end{array}\right) /\left(\mathrm{Fe}_{c} \mathrm{Co}_{1-c}\right)_{n}$ is shown [24] that also includes the case $c=1$. The results in this figure correspond to the use of a parent fcc-Cu lattice (no layer relaxation) and are supposed to illustrate the applicability and usefulness of the CPA, discussed
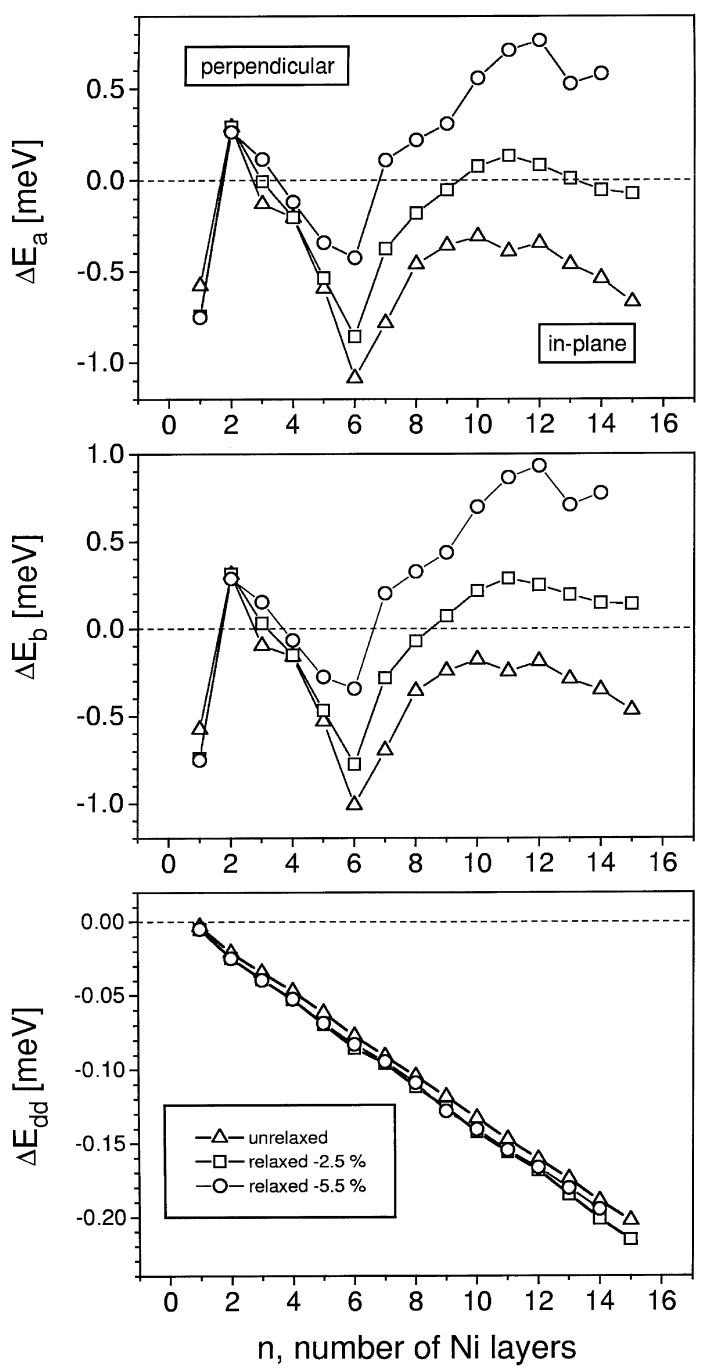

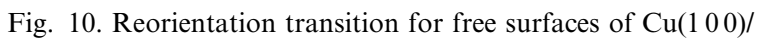
$\mathrm{Ni}_{n}$ as calculated for different interlayer spacings. Top: magnetic anisotropy energy, middle: band energy contribution, bottom: magnetic dipole-dipole contribution. From [30]. 

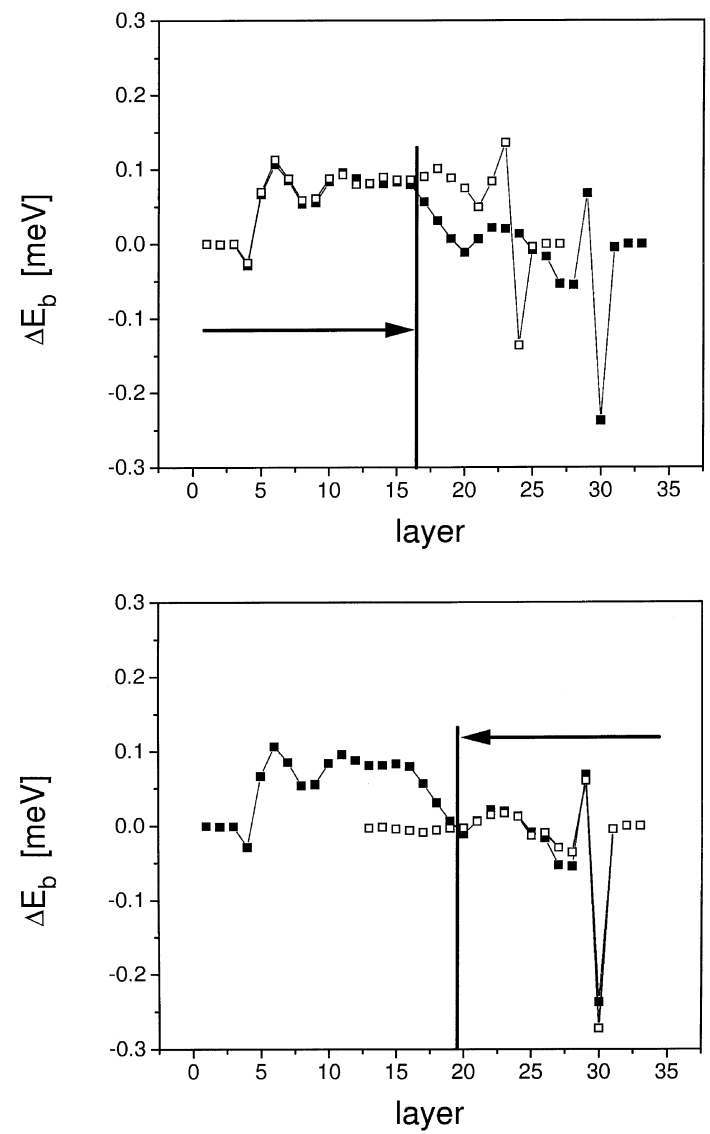

Fig. 11. A model for the band energy contribution to the magnetic anisotropy energy for free surfaces of $\mathrm{Cu}(100) / \mathrm{Ni}_{n}$ taking into account relaxation of interlayer distances and inplane spacings. Full squares: $\mathrm{Cu}(100) / \mathrm{Ni}_{15}(-5.5 \%) / \mathrm{Ni}_{12}(0 \%)$, empty squares: $\mathrm{Ni}(100) / \mathrm{Ni}_{18}(0 \%)$. From [32].

earlier. It should be noted that for $c<1$ the experimental analogon of Fig. 9 is rather of very similar shape, see [24] and references therein.

\subsection{3. $\mathrm{Ni} / \mathrm{Cu}(100)$ and $\mathrm{Co}_{m} / \mathrm{Ni}_{n} / \mathrm{Cu}(100)$}

While in most systems showing a reorientation transition this transition is from a perpendicular to an in-plane orientation of the magnetization, multilayers of $\mathrm{Ni}$ on $\mathrm{Cu}(100)$ exhibit just the opposite behavior: below about seven monolayers of $\mathrm{Ni}$ the orientation of the magnetization is in-plane, then switches with an increasing number of $\mathrm{Ni}$ layers to perpendicular (first reorientation transition), and eventually for $n \geqslant 35$ turns again to inplane (second reorientation transition). It was

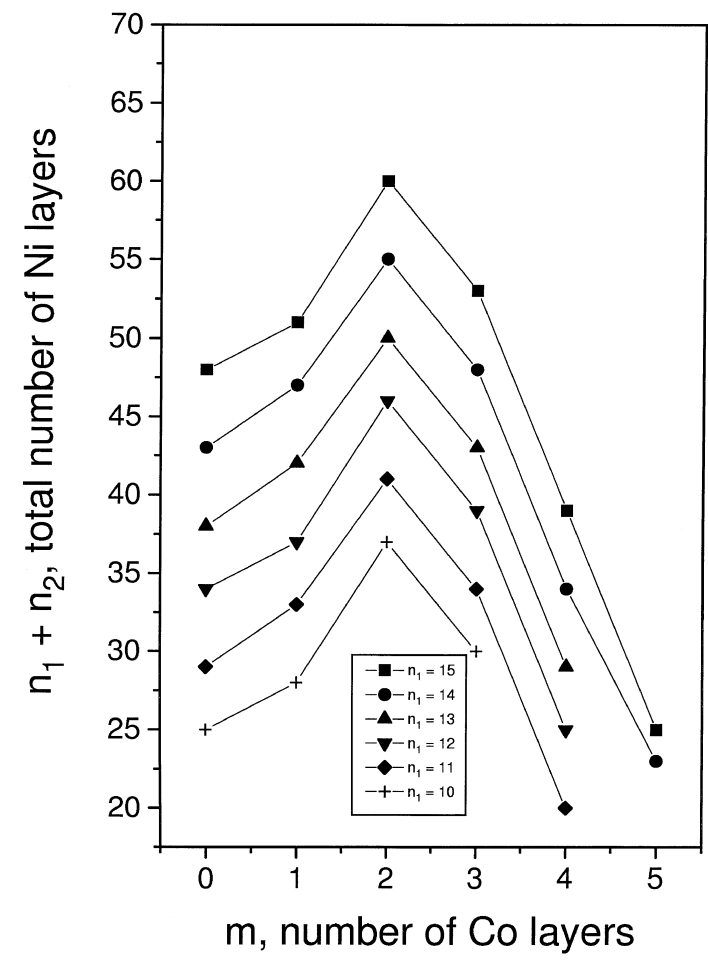

Fig. 12. Number of Co layers at which the (second) reorientation transition in relaxed free surfaces of $\mathrm{Cu}\left(\begin{array}{lll}1 & 0 & 0\end{array}\right) / \mathrm{Ni}_{\left(n_{1}+n_{2}\right)} /$ $\mathrm{Co}_{m}$ occurs. From [32].

shown recently, see [30] and the discussion therein, that the first reorientation transition is essentially caused by tetragonal distortions with respect to the lattice spacing of the substrate, since with decreasing interlayer distance the band energy contribution is considerably increased. Exactly this is shown in Fig. 10. It should be noted that experimentally the first reorientation transition occurs at about seven monolayers of $\mathrm{Ni}$, the interlayer distances being contracted by $5.5 \%$ as compared to fcc $\mathrm{Cu}$.

The second reorientation transition is even more complicated as with an increasing number of Ni layers in-plane relaxations also occur. Since this implies that no longer it can be assumed that in all atomic planes one and the same two-dimensional lattice applies, only model-like studies are at present available. Combining systems with

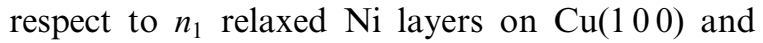
$n_{2}$ unrelaxed $\mathrm{Ni}$ layers on $\mathrm{Ni}(100)$, these two 
systems can be considered to be "stacked together", see Fig. 11, in order to predict also the second reorientation transition in the system $\mathrm{Cu}(100) / \mathrm{Ni}_{n}, n=n_{1}+n_{2}$. Fig. 12 shows the (total) number of Ni layers at which this reorientation transition occurs as a function of the thickness of a Co cap.
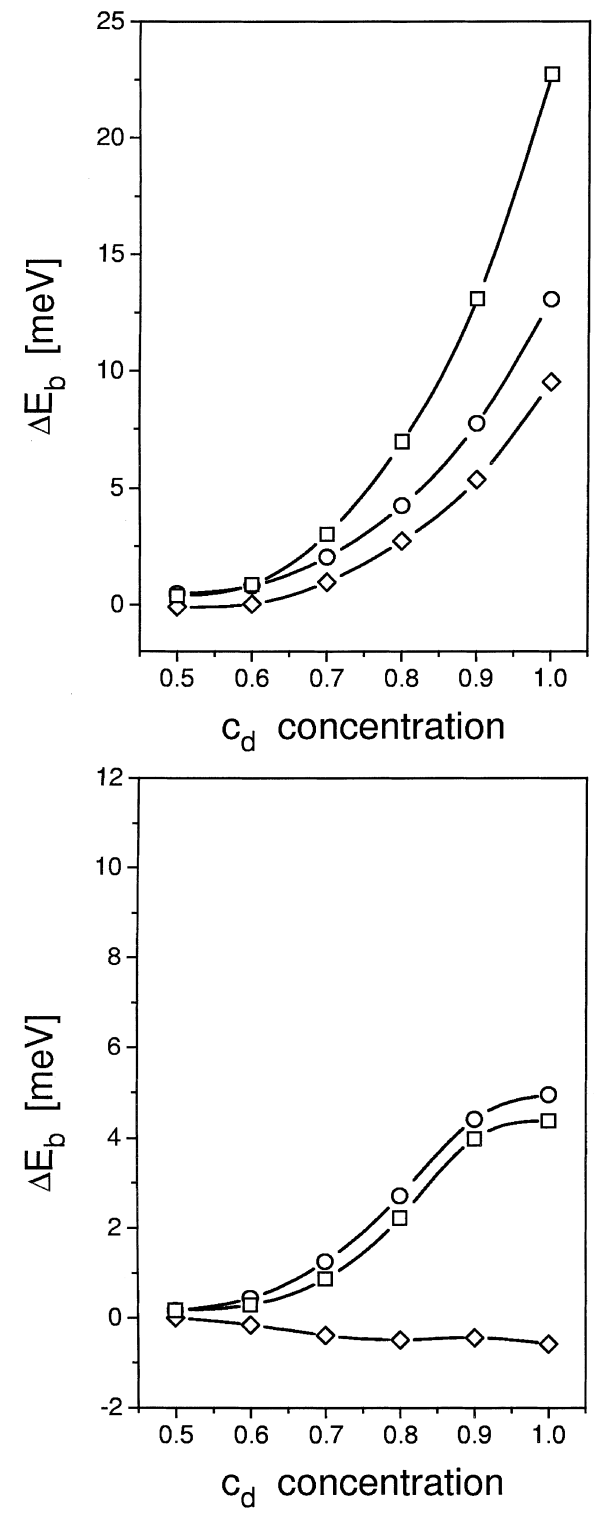

\subsection{Interdiffusion of superstructures}

$\left(\mathrm{Co}_{n} \mathrm{Pd}_{m}\right)_{r}$ superstructures on Pd-substrates or $\left(\mathrm{Co}_{n} \mathrm{Pt}_{m}\right)_{r}$ superstructures on $\mathrm{Pt}$ substrates are the technological basis of most magneto-optical devices. As the size of the (linear) Kerr effect is usually considered to be proportional to the size of
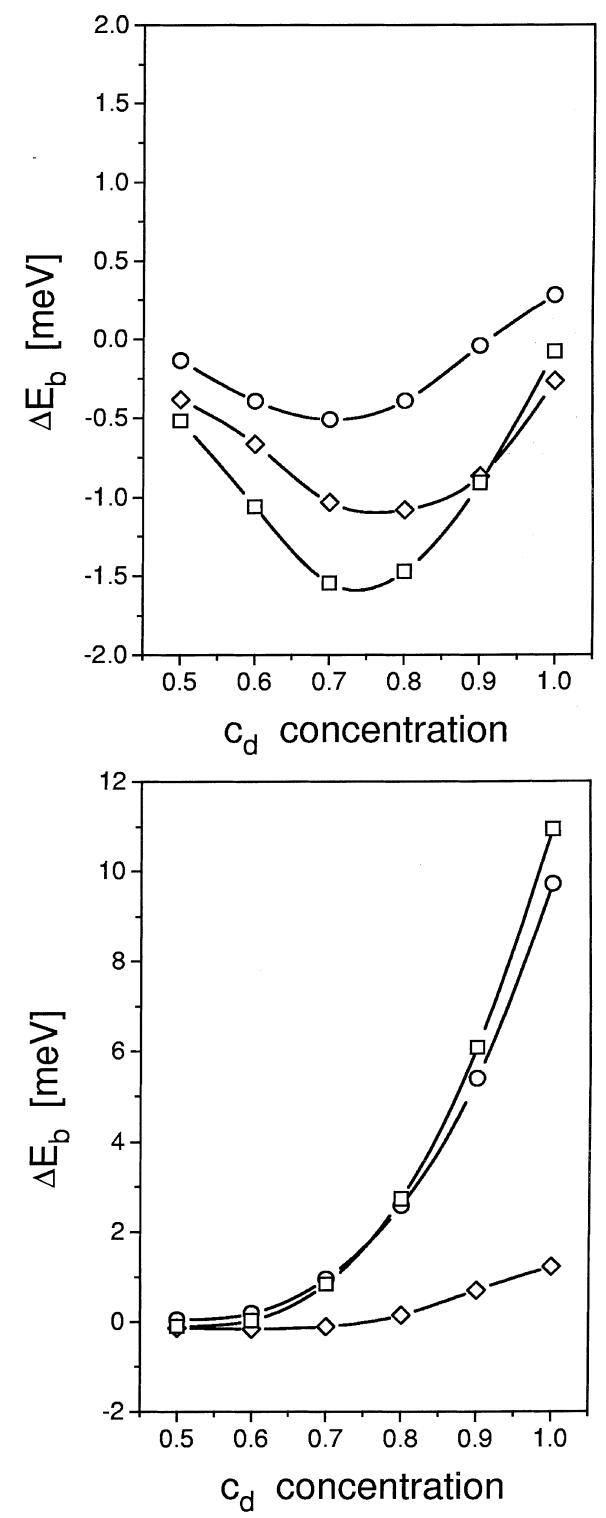

Fig. 13. Band energy contributions to the magnetic anisotropy energy in interdiffused $\left(\mathrm{Fe}_{n} \mathrm{Pt}_{n}\right)_{r}$ - and $\left(\mathrm{Co}_{n} \mathrm{Pt}_{n}\right)_{r}$-superstructures on

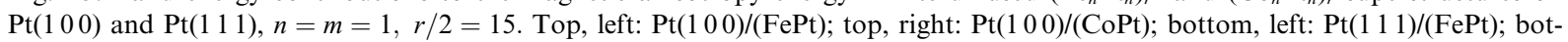

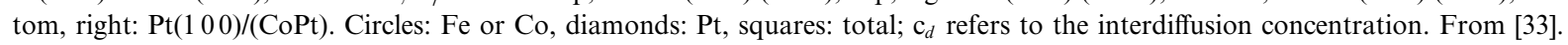


Table 1

Key of references to studies of perpendicular magnetism using the fully relativistic spin-polarized Screened KKR method

\begin{tabular}{|c|c|c|c|c|c|}
\hline Substrate & System & & & & \\
\hline $\mathrm{Cu}\left(\begin{array}{lll}1 & 0 & 0\end{array}\right)$ & $\begin{array}{l}\mathrm{Fe} \\
\mathrm{Co} \\
\mathrm{Ni} \\
\mathrm{Ni} / \mathrm{Co} \\
\left(\mathrm{Fe}_{x} \mathrm{Co}_{1-x}\right)\end{array}$ & $\begin{array}{l}{[12]} \\
{[20]} \\
{[23]} \\
{[32]} \\
{[24]}\end{array}$ & $\begin{array}{l}{[16]} \\
{[22]} \\
{[30]}\end{array}$ & [18] & [26] \\
\hline $\operatorname{Ag}\left(\begin{array}{lll}1 & 0 & 0\end{array}\right)$ & $\begin{array}{l}\mathrm{Fe} \\
\text { "4d, 5d"-overlayers }\end{array}$ & $\begin{array}{l}{[25]} \\
{[8]}\end{array}$ & [27] & [29] & \\
\hline $\mathrm{Au}\left(\begin{array}{ll}1 & 0\end{array}\right)$ & $\begin{array}{l}\mathrm{Fe} \\
\text { "4d, 5d"-overlayers }\end{array}$ & $\begin{array}{l}{[7]} \\
{[8]}\end{array}$ & [10] & [11] & [19] \\
\hline $\operatorname{Au}\left(\begin{array}{lll}1 & 1 & 1\end{array}\right)$ & Co & [14] & & & \\
\hline $\operatorname{Pt}\left(\begin{array}{lll}1 & 0 & 0\end{array}\right)$ & $\begin{array}{l}\mathrm{Fe} \\
\mathrm{Co}\end{array}$ & $\begin{array}{l}{[33]} \\
{[31]}\end{array}$ & [33] & & \\
\hline $\operatorname{Pt}\left(\begin{array}{lll}1 & 1 & 1\end{array}\right)$ & $\begin{array}{l}\mathrm{Fe} \\
\mathrm{Co}\end{array}$ & $\begin{array}{l}{[33]} \\
{[31]}\end{array}$ & [33] & & \\
\hline
\end{tabular}

the corresponding perpendicular magnetic anisotropy, the question of interdiffusion in such superstructures is of quite some importance. Assuming $n=m=1$, and considering systems of the type $\operatorname{Pt}\left(\begin{array}{lll}1 & 0 & 0)\end{array} /\left(\left(F e_{c} P t_{1-c}\right) /\left(F e_{1-c} P t_{c}\right)\right)_{r} \quad\right.$ for $\quad c=0.5$ a completely disordered equi-concentrational $\mathrm{Fe} / \mathrm{Pt}$ alloy forms the magnetic multilayer system, while for $c=1$ an ordered superstructure of $(\mathrm{CoPt})$ is regained. Denoting the $\mathrm{Fe}$ concentration in odd numbers of layers by $c_{d}$ (interdiffusion concentration), then quite clearly if $r / 2$ is an odd number for $c_{d}=1$ this leads to an Fe-terminated superstructure, whereas for $r / 2$ being an even number a Ptterminated superstructure is obtained. In Fig. 13 the effect of interdiffusion for $\mathrm{Fe} / \mathrm{Pt}$ and $\mathrm{Co} / \mathrm{Pt}$ superstructures, $r / 2=15$, on $\operatorname{Pt}\left(\begin{array}{lll}1 & 0 & 0\end{array}\right)$ and $\operatorname{Pt}\left(\begin{array}{lll}1 & 1 & 1\end{array}\right)$ are shown [33]. From this figure one easily can see that superstructures with $\mathrm{Co}$ or Fe behave quite differently, just as well as a different orientation of the substrate does matter for the actual size of the magnetic anisotropy.

\subsection{Overview}

Since only very characteristic features of magnetic anisotropy energy studies in terms of the fully relativistic spin-polarized Screened KKR method were discussed above in a rather condensed man- ner, in Table 1 a complete list of references of such studies is compiled.

Quite clearly for each system listed above a very detailed analysis of the magnetic anisotropy energy can be given in terms of layer-resolved quantities. In a few cases effects of "hybridization" were also discussed using relevant parts of orientation dependent densities-of-states. Since $\Delta E_{b}$ results from a sum over layer-dependent band energy contributions, see, e.g., Eq. (105), various partial sums can serve to distinguish and estimate surface-, interface- and "volume-like" contributions to $\Delta E_{b}$, see e.g. [30]. Although such partial sums are not really well-defined, they are useful in testing phenomenological models frequently used in the interpretive part of experimental studies.

\section{Conclusion}

The fully relativistic spin-polarized Screened KKR method proved to be extremely useful for interpreting and predicting various aspects of perpendicular magnetism. Since in principle this method is of order $N$, where $N$ is the number of planes included in the intermediate region, very large systems can be treated on an ab initio-like level. Inclusion of more than 200 atomic layers, 
i.e., multilayer thicknesses up to $300-400 \AA$, open up the possibility of describing mesoscopic systems using an essentially parameter-free quantum mechanical approach. Furthermore, since - within the limits of the local density functional theory - a fully relativistic description is used, no need of discussing the validity of perturbative approaches or the singular behavior of certain operators (spinorbit interaction) arises.

No doubt, in future applications two further improvements will be of crucial importance, namely (a) a truly full-potential version of the present approach is needed, i.e., a full potential fully relativistic spin-polarized Screened KKR implementation (on the way), and (b) theoretical means to include in-plane lattice relaxations have to be developed without violating the underlying principles of two-dimensional lattice Fourier transformation. Such means can be based either on uniform displacement relaxations of type discussed years ago [1,2], or, alternatively, using Brillouin zone integrations with an additional phase factor corresponding formally to a "continuous" non-primitive translation.

Finally, it should be noted that as a genuine Green's function approach, the applied method serves also as backbone for calculations of electric (GMR, etc.) and optical (linear Kerr effect) transport in magnetic multilayer systems, and is ideally suited as basis to any kind of (angle-integrated) spin-resolved spectroscopy.

\section{Acknowledgements}

The authors are grateful to all those who have contributed to their various studies on perpendicular magnetism. Financial support has to be acknowledged from the TMR network on 'Ab initio calculations of magnetic properties of surfaces, interfaces, and multilayers' (Contract No. EMRXCT96- 0089), the Austrian Science Foundation (Contract Nos. P11626, P12146, P12352), the Austrian Minstry of Science (Contract Nos. GZ 45.384, GZ 308.941, GZ 45.420, GZ 45.422) and the Hungarian National Science Foundation (Contract Nos. OTKA T024137 and T030240).

\section{References}

[1] A. Gonis, A.J. Freeman, P. Weinberger, Phys. Rev. B 32 (1985) 7712.

[2] A. Gonis, A.J. Freeman, P. Weinberger, Phys. Rev. B 32 (1985) 7720.

[3] G. Hörmandinger, P. Weinberger, J. Phys. Cond. Mater. 4 (1992) 2185.

[4] L. Szunyogh, B. Úfalussy, P. Weinberger, J. Kollar, Phys. Rev. B 49 (1994) 2721.

[5] B. Úfalussy, L. Szunyogh, P. Weinberger, J. Kollar, ASI series E: applied sciences, in: J.S. Faulkner, R.G. Jordan (Eds.), Kluwer Academic Publishers, Dordrecht, vol. 256, p. 301 .

[6] L. Szunyogh, B. Úfalussy, P. Weinberger, J. Kollar, J. Phys. Cond. Mater. 6 (1994) 3301.

[7] L. Szunyogh, B. Úfalussy, P. Weinberger, Phys. Rev. B 51 (1995) 9552.

[8] B. Úfalussy, L. Szunyogh, P. Weinberger, Phys. Rev. B 51 (12) (1995) 836.

[9] R. Zeller, P.H. Dederichs, B. Úfalussy, L. Szunyogh, P. Weinberger, Phys. Rev. B 52 (1995) 8807.

[10] B. Úfalussy, L. Szunyogh, P. Weinberger, J. Magnetism Magnetic Mater. 156 (1996) 255.

[11] L. Szunyogh, B. Úfalussy, P. Weinberger, C. Sommers, Phys. Rev. B 54 (1996) 6430.

[12] B. Úfalussy, L. Szunyogh, P. Weinberger, Phys. Rev. B 54 (1996) 9883.

[13] P. Weinberger, P.M. Levy, J. Banhart, L. Szunyogh, B. Úfalussy, J. Phys. Cond. Mater. 8 (1996) 7677.

[14] B. Úfalussy, L. Szunyogh, P. Bruno, P. Weinberger, Phys. Rev. Lett. 77 (1996) 1805.

[15] P. Weinberger, Philos. Mag. B 75 (1997) 509-533.

[16] B. Úfalussy, L. Szunyogh, P. Weinberger, Phys. Rev. B 55 (14) (1997) 392.

[17] P. Weinberger, I. Turek, L. Szunyogh, Int. J. Quantum Chem. 63 (1997) 165-188.

[18] B. Úfalussy, L. Szunyogh, P. Weinberger, in: J. Tobin et al. (Eds.), MRS Symposium Proceedings, vol. 475, 1997, p. 207.

[19] P. Weinberger, C. Sommers, U. Pustogowa, L. Szunyogh, B. Úfalussy, J. Phys. I France 7 (1997) 1299.

[20] L. Szunyogh, B. Úfalussy, C. Blaas, U. Pustogowa, C. Sommers, P. Weinberger, Phys. Rev. B 56 (14) (1997) 036.

[21] P. Weinberger, in: J.L. Lopez-Moran (Ed.), Current Problems in Condensed Matter: Theory and Experiment, Plenum Press, New York, 1998, p. 87.

[22] L. Szunyogh, B. Úfalussy, U. Pustogowa, P. Weinberger, Phys. Rev. B 57 (1998) 8838.

[23] J. Zabloudil, C. Uiberacker, U. Pustogowa, C. Blaas, L. Szunyogh, C. Sommers, P. Weinberger, Phys. Rev. B 57 (1998) 7804.

[24] J. Zabloudil, L. Szunyogh, U. Pustogowa, P. Weinberger, Phys. Rev. B 58 (1998) 6316.

[25] C. Sommers, J. Zabloudil, C. Uiberacker, P. Weinberger, L. Szunyogh, Phys. Rev. B 58 (1998) 5539. 
[26] C. Sommers, C. Uiberacker, P. Weinberger, L. Szunyogh, Philos. Mag. B 78 (1998) 591.

[27] L. Szunyogh, J. Zabloudil, P. Weinberger, C. Sommers, Philos. Mag. B 78 (1998) 603.

[28] C. Uiberacker, L. Szunyogh, B. Úfalussy, P. Weinberger, A. Ernst, P.H. Dederichs, Philos. Mag. B 78 (1998) 423.

[29] J. Zabloudil, C. Uiberacker, U. Pustogowa, P. Weinberger, Philos. Mag. B 78 (1998) 597.

[30] C. Uiberacker, J. Zabloudil, P. Weinberger, L. Szunyogh, C. Sommers, Phys. Rev. Lett. 82 (1998) 1289.

[31] U. Pustogowa, J. Zabloudil, C. Uiberacker, C. Blaas, P. Weinberger, L. Szunyogh, C. Sommers, Phys. Rev. B 60 (1999) 414.

[32] C. Uiberacker, J. Zabloudil, P. Weinberger, L. Szunyogh, C. Sommers, Phys. Rev. B, submitted for publication.

[33] L. Szunyogh, P. Weinberger, C. Sommers, Phys. Rev. B 60 (1999) 11910.
[34] P. Lloyd, P.V. Smith, Adv. Phys. 21 (1972) 69.

[35] P. Weinberger, Electron Scattering Theory for Ordered and Disordered Matter, Clarendon Press, Oxford, 1990.

[36] I. Turek, V. Drchal, J. Kudrnovský, M. Šob, P. Weinberger, Electronic Structure of Disordered Alloys, Surfaces and Interfaces, Kluwer Academic Publishers, Dordrecht, 1997.

[37] G.H.O. Daalderop, P.J. Kelly, M.F.H. Schuurmans, F. Jansen, Phys. Rev. B 41 (1990) 11919.

[38] E. Tsymbal, J. Magnetism Magnetic Mater. 130 (1994) L6.

[39] J.M. MacLaren, S. Crampin, D.D. Vvedensky, J.B. Pendry, Phys. Rev. B 40 (1989) 12164.

[40] M. Abramowitz, I.A. Stegun (Eds.), Handbook of Mathematical Functions, Dover, New York, 1970.

[41] S.L. Altmann, P. Herzig, Point-Group Theory Tables, Clarendon Press, Oxford, 1994).

[42] H.J.F. Jansen, Phys. Rev. B 59 (1999) 4699. 